\title{
Vector-Valued and Group-Valued Gauge Integration Via Kurzweil - Henstock's Approach
}

By

Bevan Ferreira, B.A.

\author{
A thesis submitted to \\ The Faculty of Graduate Studies and Research \\ In partial fulfillment of the requirements \\ For the degree of Master of Science
}

School of Mathematics and Statistics

Ottawa-Carleton Institute for Mathematics and Statistics

Carleton University

Ottawa, Canada

January 2004 


\author{
National Library \\ of Canada \\ Acquisitions and \\ Bibliographic Services \\ 395 Wellington Street \\ Ottawa ON K1A ON4 \\ Canada
}

Bibliothèque nationale

du Canada

Acquisisitons et services bibliographiques

395 , rue Wellington Ottawa ON K1A 0N4 Canada
Your file Votre référence ISBN: 0-612-93979-0 Our file Notre référence ISBN: 0-612-93979-0
The author has granted a nonexclusive licence allowing the National Library of Canada to reproduce, loan, distribute or sell copies of this thesis in microform, paper or electronic formats.

The author retains ownership of the copyright in this thesis. Neither the thesis nor substantial extracts from it may be printed or otherwise reproduced without the author's permission.
L'auteur a accordé une licence non exclusive permettant à la Bibliothèque nationale du Canada de reproduire, prêter, distribuer ou vendre des copies de cette thèse sous la forme de microfiche/film, de reproduction sur papier ou sur format électronique.

L'auteur conserve la propriété du droit d'auteur qui protège cette thèse. $\mathrm{Ni}$ la thèse ni des extraits substantiels de celle-ci ne doivent être imprimés ou aturement reproduits sans son autorisation.
In compliance with the Canadian Privacy Act some supporting forms may have been removed from this dissertation.

While these forms may be included in the document page count, their removal does not represent any loss of content from the dissertation.
Conformément à la loi canadienne sur la protection de la vie privée, quelques formulaires secondaires ont été enlevés de ce manuscrit.

Bien que ces formulaires aient inclus dans la pagination, il n'y aura aucun contenu manquant. 
For Joanne-John, Meestah Tijn

Oubaas Krombeen, and Collo 


\begin{abstract}
This thesis begins with a brief overview of the history of the development of the theory of integration, concluding with some more recent ideas. The method of Gauge Integration as introduced by Kurzweil and Henstock is presented, as well as an introduction to the integration of non-smooth one-forms. The vector-valued case is considered in Chapter 2, with particular attention to Kurzweil-Henstock integrable functions taking values in a Banach space. The thesis concludes with the study of multiplicative integration in a general topological group.
\end{abstract}




\section{Acknowledgements}

First I would like to thank my mother Ms Barbara Hoenson for her support and encouragement along the way. My deepest gratitude and admiration goes to Somavatey Lay, someone whose grace, courage and understanding have done so much for me.

Added to this list is an old family friend Gerald Flack, of the City of Cape Town, Municipal Government. Mrs Dorothy Heidstra was also a great help and inspiration on many occasions.

Special thanks to Dr Sam Melkonian for providing me with the opportunity to continue and complete this enterprise. Thanks again. I would also like to thank Dr Cyril Garner, for giving me the opportunity to teach at Carleton, and for his support and encouragement during this time. Dr Marlene McCallum was also very helpful, with many good suggestions and ideas. A special thankyou goes to Valerie Daley for all her assistance in navigating the process. 


\section{Contents}

Chapter 1 - Integration Theory

1.1 The Theory of Integration: a Historical Perspecive 2

1.2 Gauge Integration: An Outline 11

Chapter 2 - Vector-Valued Gauge Integrals

2.1 Notation and Terminology $\quad 22$

2.2 Basic Properties of Vector-Valued Kurzweil-Henstock Integrals 24

2.3 Bochner Integrals are Kurzweil-Henstock Integrals 28

2.4 Bochner-Integrable Functions have the Saks-Henstock Property 30

2.5 Pettis-Integrable Functions are Kurzweil-henstock Integrable 34

$\begin{array}{ll}2.6 \text { Remarks } & 38\end{array}$

\section{Chapter 3 - Riemann-Type Product Integrals}

3.1 Preamble $\quad 42$

3.2 Group-Valued Integrators $\quad 47$

3.3 Primitives and Hake's Theorem 51

3.4 Integrators With Values in the Group of Invertible Elements in a Banach Algebra 53 


\section{Chapter 1}

\section{Integration Theory}

In the present chapter we begin with a very rough sketch of the historical development of integration theory, mainly following Pesin's book [Pes] and using Priestley's textbook of calculus [Pri] as a secondary source. This will shed some light on the situation and the significance of the modern theory of conditionally convergent integrals developed by Denjoy, Perron, Khinchine, Saks, Kurzweil, Henstock, McShane etc., which is the main theme of the present thesis.

Due to the signalizing work of Kurzweil [Ku1] in 1957, the earlier descriptive theory was replaced by an analytic one, which is a simple but extremely clever modification of the classical Riemann integration by using a new notion called gauges. Later it was recognized that this "gauge integration" can be performed on more general objects which may be regarded as "nonsmooth differential forms of one-variable"; see Leader [Le1]. In the present chapter we also make use of the unpublished notes of Dr. Fong [Fo2] to give a very brief description of the basics of this theory, as well as some (unpublished) major results there. With a few exceptions, all proofs in the present chapter are omitted. 


\subsection{The Theory of Integration: a Historical Perspec- tive}

Historical records indicate that, in many civilizations, due to practical necessities or merely out of curiosity, ancient investigators studied lengths, areas, and volumes of geometric objects (not necessarily rectilinear) as a "hot" area of mathematical activities. A common approach in the good old days, described below, called the method of Eudoxus (408-355BC) in Priestly [Pri; P. 165], was very much in the spirit of integration theory taught in today's senior high schools or first year universities.

Given a geometric object, mentally divide it into small rectilinear "elements" and add up all measurements of them to produce an approximate measurement of this object. This procedure often leads to other nontrivial and interesting problems in mathematics. For example, consider the problem of finding the volume enclosed by a sphere of radius $R$. Take any diameter and divide it into $2 n$ small segments of equal length $R / n$. Each of these line segments generates a circular disk perpendicular to the diameter, with $R / n$ as its thickness. Then the total volume of all these disks is equal to

$$
2 \sum_{k=1}^{n} \pi \frac{n^{2}-k^{2}}{n^{2}} \frac{R}{n} \equiv 2 \pi R\left(1-\frac{1}{n^{3}} \sum_{k=1}^{n} k^{2}\right)
$$

which leads to the mathematical problem of finding a closed form for the sum of the finite series

$$
1^{2}+2^{2}+\cdots+n^{2}
$$

This line of mathematical investigation suggests that, in some sense, integration was developed 2000 years earlier than differentiation. It seems to be natural since many practical problems solved by integration method have immediate geometric appeal, while the rate of change problems, which are dealt with by differentiation, in comparison, are less comprehensible, in view of the confusion between velocity and acceleration in the days before Galileo. After all, a young child at an early stage learns the concept of lengths and some simple rules 
to manipulate them (e.g. if a piece of wire of length $L$ is cut into two of lengths $L_{1}$ and $L_{2}$, then $L=L_{1}+L_{2}$ ), before he or she can pick up the more complicated concept of speed, which involves duration, distance and division.

This primitive method of integration was inefficient and thorny. It was often unable to produce a desirable final answer due to the problem of summing the resulting series from the method, a task which is either impossible or requires great ingenuity to perform. For example, to find the area of the sine curve between 0 and $\pi$ by using this method, one needs to know a formula for the sum

$$
\sin t+\sin 2 t+\sin 3 t+\cdots+\sin n t
$$

According to Priestley [Pri; P. 59], Pierre de Fermat (1601-1665) was the first to introduce the notion of derivative which coincides with the one in today's calculus books. He is the one who noticed that the derivative is equal to zero at a point where a local maximum or minimum is attained. The so-called "first derivative test" in calculus books should be called Fermat's principle. (Here we should distinguish this principle in differential calculus with another one of the same name, which is about trajectories of light rays in a media with nonconstant deflection index.) Priestley called Fermat the "true inventor of differential calculus" ([Pri; P. 74]). Despite this, Leibnitz and Newton are more often regarded as the two great discoverers of calculus.

The simplicity of integration has much to do with its link to differential calculus, called the fundamental theorem of calculus. With this powerful theorem, the difficult task of evaluating sums of series such as (1.1) and (1.2) can be avoided. However, in order justify the method and validate the theory, especially to prove the fundamental theorem of calculus itself, one needs a clear and rigorous definition of integration.

The method of integration described above is a special type of limiting process. Hence its exact meaning depends on a carefully written definition of limits. The modern " $\varepsilon-\delta$ definition" of the expression $\lim _{x \rightarrow a} f(x)$ is attributed to Cauchy. Using his renewed rigor in the concept of limits, Cauchy was able to make the following definition of the integral of 
a continuous function precise, as well as to supply a sound proof for its existence. According to Cauchy (1823), the integral $\int_{a}^{b} f(x) d x$ for a continuous function $f$ on $[a, b]$ is defined to be the limit of the sum

$$
S(f, \sigma)=\sum_{k=1}^{n} f\left(x_{k-1}\right)\left(x_{k}-x_{k-1}\right)
$$

as $d(\sigma) \rightarrow 0$, where $\sigma=\left\{x_{k}\right\}_{k=0}^{n}$ is a partition of $[a, b]$, with

$$
a=x_{0}<x_{1}<x_{2}<\cdots<x_{n}=b
$$

and $d(\sigma)=\max _{1 \leq k \leq n}\left\{x_{k}-x_{k-1}\right\}$. Notice that, in the $k$ th term of the sum (1.3), Cauchy chose the left-end point of the subinterval $\left[x_{k-1}, x_{k}\right]$ to evaluate the function $f$. Certainly it is not the case that Cauchy had the insight of "non-anticipation" of Ito's integration in the modern theory of stochastic differential equations, since Cauchy only considered continuous functions in his definition and his choice was only a matter of convenience.

Riemann (1867) modified Cauchy's definition by permitting the function $f$ to be evaluated at any point $t_{k}$ in the interval $\left[x_{k-1}, x_{k}\right]$ for the $k$ th term of the sum (1.3); instead of $S(f ; \sigma)$, he used

$$
S(f ; \sigma, T)=\sum_{k=1}^{n} f\left(t_{k}\right)\left(x_{k}-x_{k-1}\right) \quad\left(\text { with } T=\left\{t_{k}\right\}_{k=1}^{n}\right)
$$

which is now called a Riemann sum. This is a considerable improvement because in his definition he allowed discontinuities in the integrand $f$. He stated a sufficient condition for integrability and he used it to prove the integrability (in his sense) of the function $f$ on $[0,1]$ he defined as follows

$$
f(x)=\sum_{n=1}^{\infty} \phi(n x) / 2^{n}, \quad \text { with } \phi(u)=\min \{u-[u],(-u)-[-u]\}
$$

([u] denotes the integral part of $u$ ). This function has a countable dense set of discontinuities. Here we should mention that the function $\left.\chi_{\mathbf{Q}}\right|_{[0,1]}$ defined on $[0,1]$ is discontinuous everywhere and is not Riemann integrable. Here, $Q$ is the set of all rational numbers, $\chi_{\mathbf{Q}}$ is the characteristic function of $Q$ and $\left.\chi_{Q}\right|_{[0,1]}$ is the restriction of $\chi_{Q}$ to $[0,1]$. 
In the second half of the 19th century, prominent mathematicians such as Dirichlet, Hölder and Cantor, tried to extend the definition of integrals for functions with a small set of discontinuities. But their definitions still have the same defects as Riemann's integral, as pointed out by Volterra (1881). The theory of integration developed at that time was still considered to be substandard when it was applied to other areas, such as Fourier analysis.

The real breakthrough was Lebesgue's pioneering paper in Comptes Rendus 1901. Instead of subdividing the closed interval $[a, b]$, he subdivided the range of $f$. For the moment, assume that $f$ is bounded, say $c<f(x)<d$ for all $x \in[a, b]$. Take a partition of $[c, d]$, say

$$
\sigma: c=y_{0}<y_{1}<\cdots<y_{n}=d .
$$

For simplicity, we write $\left\{y_{k-1}<f \leq y_{k}\right\}$ for the set

$$
f^{-1}\left(\left(y_{k-1}, y_{k}\right]\right) \equiv\left\{x \in[a, b] \mid y_{k-1}<f(x) \leq y_{k}\right\}
$$

Then we should have

$$
\underline{S}_{\sigma} \equiv \sum_{k=1}^{n} y_{k-1} m\left(\left\{y_{k-1}<f \leq y_{k}\right\}\right) \leq \int_{a}^{b} f(x) d x
$$

and

$$
\int_{a}^{b} f(x) d x \leq \bar{S}_{\sigma} \equiv \sum_{k=1}^{n} y_{k} m\left(\left\{y_{k-1}<f \leq y_{k}\right\}\right)
$$

where $m$ stands for some "measure". Notice that

$$
0 \leq \bar{S}_{\sigma}-\underline{S}_{\sigma} \leq r(\sigma)(b-a)
$$

where $r(\sigma)=\sup _{1 \leq k \leq n}\left|y_{k}-y_{k-1}\right|$. The question of getting a proper definition of integral boils down to this: how to endow a proper meaning of this "measure" $m$ ?

If $U$ is a bounded open set in $\mathbf{R}$, then $U$ is the union of a finite or countably many disjoint open intervals $I_{n}$ and its measure is defined to be the sum of the lengths of these intervals: $m(U)=\sum_{n}\left|I_{n}\right|$. If $F$ is a closed bounded set, we take any open interval $I$ containing $F$ and define the measure of $F$ by putting $m(F)=|I|-m(I \backslash F)$; (notice 
that $I \backslash F$ is an open set). For any bounded set $E$, its inner measure $m_{*}(E)$ and its outer measure $m^{*}(E)$ are defined via

$$
m_{*}(E)=\sup \{m(F): E \supseteq F, F \text { is closed }\}
$$

and

$$
m^{*}(E)=\inf \{m(U): E \subseteq U, U \text { is open }\}
$$

Using a compactness argument, it is easy to prove that $m_{*}(E) \leq m^{*}(E)$. When $m_{*}(E)$ and $m^{*}(E)$ are equal, we say that $E$ is measurable (in Lebesgue's sense), and we call their cormmon value the (Lebesgue) measure of $E$. One of the most important properties of this measure $m$ is the countable additivity: if $\left\{E_{k}\right\}_{k=1}^{\infty}$ is a countable collection of measurable sets, then their union $\bigcup_{k=1}^{\infty} E_{k}$ is also measurable, and if, in addition, the $E_{k}$ 's are mutually disjoint, then

$$
m(E)=\sum_{k=1}^{\infty} m\left(E_{k}\right)
$$

After Lebesgue's seminal paper, the study of measure and integration quickly developed into a mature theory pursued by a host of analysts, among whom we only mention E.Borel, W.H. Young, F.Riesz, C.Carathéodory and J.Radon.

Due to its power and near perfection, and its important role in probability theory, harmonic analysis, geometric integration, etc., measure theory has become a major mathematical subject in training today's analysts. Using today's standard, Riemann integration in comparison seems to be awkward, old fashioned, inefficient and almost useless. According to Dieudonné, it is a 'theory' that has at best the importance of a mildly interesting exercise in the general theory of measure and integration. However, one cannot get rid of Riemann-type integrals entirely. For example, the most common way of defining an Ito integral $I_{f}(\omega) \equiv \int_{0}^{T} f(t, \omega) d B_{t}(\omega)$ is to use the Riemann sums

$$
S_{\sigma}(\omega) \equiv \sum_{k=1}^{n} f\left(t_{k-1}, \omega\right)\left(B_{t_{k}}(\omega)-B_{t_{k-1}}(\omega)\right)
$$

which can be considered as the Ito integral of the elementary function

$$
f_{\sigma}(t, \omega) \equiv \sum_{k=1}^{n} f\left(t_{k-1}, \omega\right) \chi\left[t_{k-1}, t_{k}\right)(\omega)
$$


It turns out that we have the following identity

$$
\mathbf{E}\left|\int_{0}^{T} f_{\sigma}(t, \cdot) d B_{t}\right|^{2}=\mathbf{E} \int_{0}^{T}\left|f_{\sigma}(t, \cdot)\right|^{2} d t
$$

called the Ito isometry, where $\mathbf{E}$ stands for the expectation of a random variable. The Ito integral $I_{f}(\omega)$ can then be defined in an elementary way: extension by isometry; see Okesendal [Oke; P.26] for details. Another expression defined by Riemann type integration is the triangular truncation of a compact operator $K$ on a Hilbert space $H: \int_{\mathcal{C}} P_{M} K d P_{M}$, where $\mathcal{C}$ is a closed chain of (closed) subspaces $M$ of $H$ and $P_{M}$ is the orthogonal projection onto $M$; see [GoK; P.41].

Let $f$ be a Lebesgue integrable function on $[a, b]$ and let $F$ be its primitive:

$$
F(x)=\int_{a}^{x} f(t) d t, \quad a \leq x \leq b .
$$

Then we have:

1. $F$ is continuous with $F(a)=0$,

2. $F$ is differentiable almost everywhere, and

3. $F$ is absolutely continuous.

Conversely, if $F$ is a function on $[a, b]$ satisfying all three conditions above, then $F$ is the primitive of some Lebesgue integrable function $f$ and in fact we can take $f$ to be $F^{\prime}$. (The proofs of these facts are in any standard textbook of measure theory.) A natural question is, can condition 3 be replaced by some weaker condition so that $F$ is uniquely determined by $f$ ? If $\mathcal{P}$ stands for some linear space of functions satisfying the first two conditions plus a weaker third condition such that, for all $F, G \in \mathcal{P}, F^{\prime}=G^{\prime}$ almost everywhere, then we can extend the Lebesgue integration in the following way. Let $\mathcal{C}=\left\{F^{\prime} \mid F \in \mathcal{P}\right\}$. We define the integral of any $f \in \mathcal{C}$ by putting $\int_{a}^{b} f=F(b)-F(a)$, where $F$ is a function in $\mathcal{P}$ satisfying $F^{\prime}=f$ almost everywhere. The definition of integration of functions in class $\mathcal{C}$ here is called a descriptive definition. This approach 
of extending Lebesgue integration is natural since $F$ can be considered to be a solution of the differential equation $d y=f(x) d x$ with the initial condition $y(a)=0$ and the question we are considering is essentially about the existence and uniqueness of its solution. That condition 3 cannot be entirely discarded was vividly illustrated by a theorem of Luzin (1917): given any measurable function $f$ on $[a, b]$, there exists a continuous function $F$ on $[a, b]$ such that $F^{\prime}=f$ almost everywhere;[Sak; P.217]. Of course not all measurable functions are Lebesgue integrable.

Lebesgue recognized this problem of searching for an appropriate descriptive definition to extend his theory in his 1902 Compte Rendus paper. He noticed that condition 3 for integrability in his sense cannot be discarded, even when condition 2 is strengthened to existence of the derivative $F^{\prime}(x)$ for every $x$. An easy example is

$$
F(x)=\frac{1}{2} x^{2} \sin \frac{1}{x^{2}} .
$$

Then $F^{\prime}(x)=x \sin \left(1 / x^{2}\right)-(1 / x) \cos \left(1 / x^{2}\right)$ for $x \neq 0$ and $F^{\prime}(0)=0$. One can check that $\int_{0}^{1}\left|F^{\prime}(x)\right| d x=\infty$ and hence $F^{\prime}$ is not Lebesgue integrable. Actually, Lebesgue gave a more complicated example to suggest a problem for future research. In 1912, Denjoy, following a transfinite procedure based on Lebesgue's idea, introduced a new integration, to which Khinchine also made substantial contribution. Then Perron also introduced a new integration based on a completely different idea called majorization in 1914. About ten years later, Hake, Alexandroff and Looman independently proved that Denjoy's integration is equivalent to Perron's integration. Here we follow Saks [Sak] to give a descriptive definition of (restricted) Denjoy integrals.

Let $F$ be a continuous function on $[a, b]$. For each subinterval $I$ of $[a, b]$, we denote by $\mathrm{O}_{F}(I)$ the oscillation of $F$ over $I$ :

$$
\mathrm{O}_{F}(I)=\sup _{u, v \in I}|F(u)-F(v)| .
$$

For any subset $A$ of $[a, b]$, We say that $F$ is of bounded variation in the strict sense (or simply $\mathrm{BV}_{*}$ ) on $A$ if there exists $M>0$ such that $\sum_{k=1}^{N} \mathrm{O}_{F}\left(I_{k}\right) \leq M$ for all 
nonoverlapping subintervals $I_{k}(1 \leq k \leq N)$ with their end points in $A$. We say that $F$ is of generalized bounded variation in the strict sense, or in the class $B G_{*}$, if we can write $[a, b]$ as a countable union of sets $\bigcup_{n=1}^{\infty} A_{n}$ such that $F$ is $B V_{*}$ on each $A_{n}$. (In [Fo2], such $F$ is called a function of $\sigma$-bounded variation.) We can define generalized absolute continuity in the strict sense, or $\mathrm{ACG}_{*}$, in the same manner. It turns out that, if $F$ is of class $\mathrm{ACG}_{*}$ and if $F^{\prime}=0$ almost everywhere, then $F$ is a constant function; see Saks [Sak; Theorem 6.2 on P.225]. Thus the class $A_{C G}$ admits a descriptive definition of integration, which extends Lebesgue's theory.

In 1957, to study the existence theorem of initial-value problems in ordinary differential equations, Kurzweil [Ku1] introduced what is known as gauge integration or KurzweilHenstock integration today. It is a simple but extremely clever modification of Riemann integration. One may claim that Kurzweil's approach revived Riemann's theory, saving it from being a dead subject. However, here we should mention that the techniques employed in developing this new theory are very different from those used in the traditional Riemann integration. To be fair, here we also mention two of the other pioneering workers: Henstock [Hel] and McShane [Mc1].

By a gauge on $[a, b]$ we simply mean a function $\gamma$ on $[a, b]$ such that $\gamma(t)>0$ for all $t \in[a, b]$. We say that a function $f$ on $[a, b]$ is gauge integrable, or Kurzweil-Henstock integrable, with $\int_{a}^{b} f(x) d x=I$, if, given $\varepsilon>0$, there exists a gauge $\gamma$ such that, for all partitions $\sigma=\left\{x_{k}\right\}_{k=1}^{N}$ of $[a, b]$ with a set of "tags" $T=\left\{t_{k}\right\}_{k=1}^{N}$ such that $x_{k-1} \leq t_{k} \leq x_{k}$ and $\max \left\{t_{k}-x_{k-1}, x_{k}-t_{k}\right\}<\gamma\left(t_{k}\right)$, that is,

$$
\left[x_{k-1}, x_{k}\right] \subseteq\left(t_{k}-\gamma\left(t_{k}\right), t_{k}+\gamma\left(t_{k}\right)\right)
$$

for all $k$ with $1 \leq k \leq N$, we have $|S(f ; \sigma, T)-I|<\varepsilon$, where $S(f ; \sigma, T)$ is given by (1.4) above. A partition $\sigma$ with tags $T$ such as the one given here is said to be $\gamma$-fine if (1.5) holds for all $k$.

In order to demonstrate how this definition works, we show that the function $g \equiv \chi_{\mathrm{Q}} \mid\{0,1]$ (which is not Riemman integrable) is integrable in Kurzweil's sense with $\int_{0}^{1} g=0$. Put 
all rational numbers between 0 and 1 into a sequence, say $\left\{r_{n}\right\}_{n=1}^{\infty}$. Let $\varepsilon>0$ be given. Define a gauge by putting $\gamma\left(r_{k}\right)=\varepsilon / 2^{k+1}$ and $\gamma(t)=1$ if $t$ is irrational. Then it is easy to check that $S(g ; \sigma, T)<\varepsilon$ for all $\gamma$-fine tagged divisions $(\sigma, T)$. There are many advantages of gauge integration over Lebesgue integration, but we only mention two here. First, it is more general; in fact, it includes both Lebesgue integrals and improper integrals. Second, the fundamental theorem of calculus has an improved version, which follows from the definition almost immediately.

In 1973, McShane dropped the requirement $x_{k-1} \leq t_{k} \leq x_{k}$, but (1.5) is still in force. The resulting integrals, now called McShane's integrals, turn out to be equivalent to Lebesgue integrals.

There are attempts to extend gauge integration and apply it to stochastic integrals and Feymann integrals. But it seems that such attempts do not meet with great success in attracting the attention of probabilists or physicists. Strengthening its relation with other mathematical areas and extending it to new directions such as multi-dimensional theory in order to assert a role in geometric measure theory, still require substantial efforts in the future to make this subject "respectable".

\subsection{Theory of Gauge Integration: An Outline}

In the current section we summarize basic definitions and results about gauge integration according to the unpublished notes [Fo2].

Before we begin, let us say something about the justification for terminology here, according to a conversation with Dr.Fong. The word "totalization" was quite common for new types of integration in the early 20th century, especially in Denjoy's papers. This word borrowed from the old literature is good for our purposes because it often conveys the right idea and the correct sense. For example, we will see that an integrator is totalizable if and only if it is (totally) equivalent to a total differential $d F$. The words "integrator" and "integrand" were occasionally used in the past - the latter is still in use today - and they appear for ex- 
ample in the chapter about Riemman-Stieltjes integrals in Tom Apostal's Calculus textbook [Apo]. These two obsolete words were "recycled" in [Fo2]. The word "figure" appeared in Saks classic [Sak] and now it is a standard word used in the gauge integration community. This word suggests the usage of "style" instead of "type" in the notes. The reason to avoid the word "type" used in this way is because of the frequent appearance of the expression "Riemann-type integrals", which may lead to conflicts and confusion.

Denote by $\mathcal{I}$ the set of all closed intervals in $\mathrm{R}$. By a style we mean a subset $\mathcal{S}$ of $\mathbf{R} \times \mathcal{I}$. An element $(t, I)$ in $\mathcal{S}$ is called a tagged interval of style $\mathcal{S}$. A collection $\mathcal{F}^{T}=\left\{\left(t_{j}, I_{j}\right)\right\}_{j=1}^{N}$ of non-overlapping tagged intervals of style $\mathcal{S}$ is called a (tagged) figure in style $\mathcal{S}$. Given a gauge $\gamma$ on $[a, b]$, we say that the figure $\mathcal{F}$ is $\gamma$-fine if $I_{j} \subseteq\left(t_{j}-\gamma\left(t_{j}\right), t_{j}+\gamma\left(t_{j}\right)\right)$ for all $j$. In case $[a, b]=\bigcup_{j=1}^{N} I_{j}$, we call $\mathcal{F}^{T}$ a tagged partition of $[a, b]$. We say that a style $\mathcal{S}$ has Cousin's property if, given any gauge $\gamma$ on $[a, b]$, there is a $\gamma$-fine tagged partition of $[a, b]$ in style $\mathcal{S}$. We are mainly interested in two styles:

$$
\mathcal{S}_{\mathrm{bd}}=\{(t, I) \in \mathbf{R} \times \mathcal{I} \mid t \in I\}
$$

called the bound style since $t$ is bound to $I$ for a tagged interval $(t, I)$ in this style, and

$$
\mathcal{S}_{\max }=\mathrm{R} \times \mathcal{I}
$$

called the free style since it has the maximum freedom in picking tagged intervals. The bound style is used in Kurzweil-Henstock integration and the free style is used for McShane integration and the Hadamard derivative. Both the bound style and the free style have Cousin's property. In view of the way Ito's integral is defined, we may entertain the idea of introducing the "left style" $\mathcal{S}_{\ell}=\{(t,[u, v]) \in \mathrm{R} \times \mathcal{I} \mid u=t\}$. Unfortunately $\mathcal{S}_{\ell}$ does not have Cousin's property.

By an integrator in the style $\mathcal{S}$ we mean a map $\alpha: \mathcal{S} \rightarrow \mathrm{R}$. (We wish to call $\alpha$ a nonsmooth differential form of one variable, but that name is too long.) Given a tagged partition or a figure $\mathcal{F}^{T}=\left\{\left(t_{j}, I_{j}\right)\right\}_{j=1}^{N}$, the corresponding "Riemman sum" $S\left(\alpha ; \mathcal{F}^{T}\right)$ is defined to be $\sum_{j=1}^{N} \alpha\left(t_{j}, I_{j}\right)$. Given a gauge $\gamma$ on $[a, b]$, denote by

$$
\mathcal{S}_{a}^{b}(\alpha, \gamma)(\equiv \mathcal{S}(\alpha, \gamma) \equiv \mathcal{S}(\gamma))
$$


the set of all Riemann sums $S\left(\alpha, \mathcal{P}^{T}\right)$, where $\mathcal{P}^{T}$ are $\gamma$-fine tagged partitions. We say that an integrator $\alpha$ is totalizable and its total $\int_{a}^{b} \alpha$ is equal to $A$ if, given $\varepsilon>0$, there exists a gauge $\gamma$ on $[a, b]$ such that $\mathcal{S}_{a}^{b}(\alpha, \gamma) \subseteq(A-\varepsilon, A+\varepsilon)$. Certainly, in order for this definition to make sense, we have to assume that the style $\mathcal{S}$ we use here has Cousin's property. So, in the rest of the thesis, we make the blanket assumption that all styles have Cousin's property.

Notice that the set $\Gamma \equiv \Gamma_{[a, b]}$ of all gauges on $[a, b]$ is directed downward and hence it is possible to consider the net $\mathcal{D}_{\alpha}=\left\{\operatorname{diam}(\mathcal{S}(\alpha, \gamma)\}_{\gamma \in \Gamma}\right.$, where diam stands for the diameter of a set: $\operatorname{diam}(S)=\sup _{x, y \in S}|x-y|$. Using Cauchy's criterion for convergence, it is easy to show that $\alpha$ is totalizable if and only if the net $\mathcal{D}_{\alpha}$ tends to zero; (we will call this Cauchy's criterion for totalization). Furthermore, we define the upper and lower "Darboux sums" $\bar{S}(\gamma)$ and $\underline{S}(\gamma)$ by putting

$$
\bar{S}(\gamma)=\sup \mathcal{S}(\alpha, \gamma), \quad \underline{S}(\gamma)=\inf \mathcal{S}(\alpha, \gamma)
$$

and the upper and lower totals $\bar{\int}_{a}^{b} \alpha$ and $\int_{-a}^{b} \alpha$ by putting

$$
\bar{\int}_{a}^{b} \alpha=\lim _{\gamma \in \Gamma} \bar{S}(\gamma) \equiv \sup _{\gamma} \bar{S}(\gamma), \quad \int_{a}^{b} \alpha=\lim _{\gamma \in \Gamma} \underline{S}(\gamma) \equiv \inf _{\gamma} \underline{S}(\gamma) .
$$

Then it is easy to check that $\alpha$ is totalizable if and only if

$$
-\infty<\int_{a}^{b} \alpha=\bar{\int}_{a}^{b} \alpha<+\infty .
$$

Given any function $F$ on $[a, b]$, the total differential $d F$ is the integrator defined by putting $d F(t,[u, v])=F(v)-F(u)$. It is clear that $d F$ is always totalizable with $\int_{a}^{b} d F=F(b)-F(a)$. Given a function $f$ and a finite Borel measure $\mu$ on $[a, b], f d \mu$ is the integrator defined by putting $f d \mu(t, I)=f(t) \mu(I)$. Notice that $d \mu$ is totalizable and $\int_{a}^{b} d \mu=\mu[a, b]$. When $\mu$ is the Lebesgue measure, we write $\int d x$ for $f d \mu$ and $\int_{a}^{b} f$ for $\int_{a}^{b} f d \mu$. When the style we use is $\mathcal{S}_{\mathrm{bd}}$, the corresponding totalization is called Kurzweil-Henstock integration. When the style we use is the free style, the corresponding totalization is called McShane integration. 
We have the following elementary properties of totalization: (a) if $\alpha$ is totalizable over $[a, b]$, then it is also totalizable over any subinterval of $[a, b]$; (b) if $a<c<b$, and if $\alpha$ is totalizable over $[a, b]$ and is atomless at $c$, then $\int_{a}^{b} \alpha=\int_{a}^{c} \alpha+\int_{c}^{b} \alpha$, where we say that $\alpha$ is atomless at $c$ means that $\lim _{|I| \rightarrow 0} \alpha(c, I)=0$; (c) if $a<c<b$, if $\alpha$ is totalizable over both $[a, c]$ and $[c, b]$ and if $\alpha$ is atomless at $c$, then $\alpha$ is totalizable over $[a, b]$; (d) if $\alpha$ and $\beta$ are totalizable over $[a, b]$ and $p$ and $q$ are constants, then $p \alpha+q \beta$ is totalizable and $\int_{a}^{b}(p \alpha+q \beta)=p \int_{a}^{b} \alpha+q \int_{a}^{b} \beta$. It is assumed that integrators here are of the same style (normally this assumption is clear from the context and we prefer not to mention it explicitly). Following the general policy of the present section, we skip the proofs of these properties. However we mention that, for proving part (a), we use Cauchy's criterion for totalization.

The so called Saks-Henstock's lemma, stated in the form below, may be regarded as a technical triumph of gauge integration:

THEOREM 1.1 (Saks-Henstock's lemma) If $\alpha$ is a totalizable integrator of style $\mathcal{S}$ over $[a, b], \varepsilon$ is a positive number and $\gamma$ is a gauge on $[a, b]$ such that $\mathcal{S}(\alpha, \gamma)$ is within $\varepsilon / 4$ of $\int_{a}^{b} \alpha$, then, for each $\gamma$-fine tagged figure $\left\{\left(t_{j}, I_{j}\right)\right\}_{j=1}^{N}$ in style $\mathcal{S}$, we have

$$
\sum_{j=1}^{N}\left|\alpha\left(t_{j}, I_{j}\right)-\int_{I_{j}} \alpha\right|<\varepsilon
$$

We say that two integrators $\alpha$ and $\beta$ of style $\mathcal{S}$ on $[a, b]$ are totally equivalent over a set $S$ if, for each $\varepsilon>0$, there exists a gauge $\gamma$ on $[a, b]$ such that

$$
\sum_{k=1}^{N}\left|\alpha\left(t_{k}, I_{k}\right)-\beta\left(t_{k}, I_{k}\right)\right|<\varepsilon
$$

for all $\gamma$-fine figures $\left\{\left(t_{j}, I_{j}\right)\right\}_{j=1}^{N}$ of style $\mathcal{S}$ anchored to $S$, that is, $t_{k} \in S$ for all $k$. When $S=[a, b]$, we simply say that $\alpha$ and $\beta$ are totally equivalent. Using Saks-Henstock's lemma, it is easy to establish:

1. An integrator $\alpha$ is totalizable if and only if it is totally equivalent to a total differential, namely, $d F$ for some function $F$. 
2. Two totalizable integrators $\alpha$ and $\beta$ on $[a, b]$ are totally equivalent if and only if $\int_{u}^{v} \alpha=\int_{u}^{v} \beta$ for all subintervals $[u, v]$ of $[a, b]$.

3. If $\alpha$ is totalizable over $[a, b]$ and if $F(x)=\int_{a}^{x} \alpha(a \leq x \leq b)$, then $F$ is continuous and $\alpha$ is totally equivalent to $d F$.

With the integrator $f \alpha$ defined by $(f \alpha)(t, I)=f(t) \alpha(t, I)$ for $f$ a function on $[a, b]$, the following lemma gives the basic property of total equivalence:

Lemma 1.2 Let $\alpha$ and $\beta$ be integrators on $[a, b]$. (a) If $\alpha$ and $\beta$ are totally equivalent on a subset $S_{n}$ of $[a, b]$ for each $n=1,2, \ldots$, then they are totally equivalent on $S=\bigcup_{n=1}^{\infty} S_{n}$. (b) If $\alpha$ and $\beta$ are totally equivalent and $f$ is any function on $[a, b]$, then $f \alpha$ and $f \beta$ are also totally equivalent.

Here, $f \alpha$ is the integrator defined by $(f \alpha)(t, I)=f(t) \alpha(t, I)$. We make an exception here by proving this lemma, because the proof is short and because it vividly illustrates a common technique in gauge integration called "splicing". We assume that $\beta=0$ since we may consider $\alpha-\beta$ instead. Since total equivalence on $S$ implies total equivalence on any subset of $S$, in (a) we may assume that $S_{n}$ are mutually disjoint. Let $\varepsilon>0$ be given. Then, for each $n$, there is a gauge $\gamma_{n}$ such that

$$
S\left(|\alpha|, \mathcal{F}^{T}\right) \equiv \sum_{k=1}^{N}\left|\alpha\left(t_{k}, I_{k}\right)\right|<\varepsilon / 2^{n+1}
$$

for all $\gamma_{n}$-fine tagged figure $\mathcal{F}^{T}=\left\{\left(t_{k}, I_{k}\right)\right\}_{k=1}^{N}$ anchored to $S_{n}$. Define a gauge $\gamma$ on $[a, b]$ by putting $\gamma(t)=\gamma_{n}(t)$ if $t \in S_{n}$ for any $n$ and $\gamma(t)=1$ otherwise. Then it is easy to check that $S\left(|\alpha|, \mathcal{F}^{T}\right)<\varepsilon$ for every tagged figure $\mathcal{F}^{T}$ anchored to $S$. To prove (b), first we quickly check that $f \alpha$ is totally equivalent to 0 on

$$
S_{n}=\left\{t \in[a, b]|| f_{n}(t) \mid \leq n\right\}
$$

Notice that $\bigcup_{n=1}^{\infty} S_{n}=[a, b]$ and hence (b) follows from (a).

Using the above lemma, we can give a short proof of Hake's theorem; we obtained this proof from Leader [Le2]. This theorem tells us that Kurzweil-Henstock integration includes 
improper integrals and hence is "more powerful" (words used by Bartle) than Lebesgue integration. The standard proof of this theorem $([\mathrm{Pfe}])$ is rather complicated and technical. In Chapter 3 we will prove a group-valued version of Hake's theorem, using the line of argument in this stanard proof.

Corollary 1.3 (Hake's Theorem) If $f$ on $[a, b]$ is Kurzweil-Henstock integrable over $[a, c]$ for each $c$ with $a<c<b$; and $L \equiv \lim _{c \uparrow b} \int_{a}^{c} f$ exists, then $f$ is Kurzweil-Henstock integrable over $[a, b]$.

To prove this, take any sequence $\left\{c_{n}\right\}$ in $(a, b)$ increasing to $b$. Let $S_{n}=\left[a, c_{n}\right]$ and $B=\{b\}$. Define the continuous function $F$ on $[a, b]$ by $F(x)=\int_{a}^{x} f$ for $x<b$ and $F(b)=L$. Then it is clear that $\alpha$ is totally equivalent to $d F$ on each $S_{n}$. Clearly $\alpha$ is totally equivalent to 0 on $B$. That $d F$ is totally equivalent to 0 on $B$ depends on the style $\mathcal{S}_{\mathrm{bd}}$ we choose here: a tagged figure of this style anchored on a singleton set consists of no more than two tagged intervals. (This is not true when the free style $\mathcal{S}_{\max }$ is chosen. In fact, Hake's theorem fails in this case.)

The following variant of Saks-Henstock's is crucial for studying local variations of functions and upper totals of functions with respect to a positive integrator. First a definition: we say that an integrator $\alpha$ is totally bounded above if there is a gauge $\gamma$ such that $\mathcal{S}(\alpha, \gamma)$ is bounded above. In that case, $\bar{\int}_{a}^{b} \alpha<+\infty$.

THEOREM 1.4 If $\alpha$ is an integrator that is totally bounded above such that $\bar{\int}_{a}^{b} \alpha>-\infty$, then, given $\varepsilon>0$, there is a gauge $\gamma$ such that

$$
\sum_{j=1}^{N}\left(\alpha\left(t_{j}, I_{j}\right)-\bar{J}_{I_{j}} \alpha\right)<\varepsilon
$$

for all $\gamma$-fine figures $\left\{\left(t_{j}, I_{j}\right)\right\}_{j=1}^{N}$.

Observe that no absolute values are used in the statement of this proof. This is necessary as the corresponding statement using absolute values fails to be true. 
We say that an integrator $\omega$ of style $\mathcal{S}$ is positive if $\omega(t, I) \geq 0$ for all tagged intervals $(t, I)$ in $\mathcal{S}$. Using the technique of splicing, together with Saks-Henstock's lemma, we can establish

Proposition 1.5 Given a positive integrator $\omega$, the collection $\mathcal{R}_{\omega}$ of all subsets $A$ such that $\chi_{A} \omega$ is totalizable is a $\sigma$-ring.

That $\mathcal{R}_{\omega}$ a $\sigma$-ring here we mean $A, B \in \mathcal{R}_{\omega}$ entails $A \cup B, A \backslash B \in \mathcal{R}_{\omega}$ and $A_{n} \in \mathcal{R}_{\omega}$ with $\sum_{n=1}^{\infty} \int_{a}^{b} \chi_{A_{n}} \omega<+\infty$ entails $\bigcup_{n=1}^{\infty} A_{n} \in \mathcal{R}_{\omega}$. A difficult problem concerns the inner regularity of sets in $\mathcal{R}_{\omega}$ due to the possibility that no open set is in $\mathcal{R}_{\omega}$ except the empty set; for example, this occurrs when $\omega$ is given by $\omega(t, I)=|I|^{p}$, where $0<p<1$ is fixed. This sort of difficulty was clearly pointed out by Rogers [Rog; P.36] in dealing with infinite measures.

However, when $\omega$ is finite or $\sigma$-finite, the situation is pretty well understood. We say that $\omega$ is finite if it is totalizable.

Proposition 1.6 If $\omega$ is a totalizable positive integrator on $[a, b]$, then $\mathcal{R}_{\omega}$ is a $\sigma-$ algebra containing all open subsets of $[a, b]$, and the set function $\mu_{\omega}$ on $\mathcal{R}_{\omega}$ given by $\mu_{\omega}(A)=\int_{a}^{b} \chi_{A} \omega$ is a finite measure.

Next we consider totalization of the expression $\int_{a}^{b} f \omega$, where $f$ is a function on $[a, b]$ and $\omega$ is a totalizable positive integrator without atoms. (Sometimes we call $f$ an integrand.) When the style is $\mathcal{S}_{\max }$, we say $f \omega$ is McShane integrable if it is totalizable (with respect to the free style). We have the following version of McShane's theorem:

THEOREM 1.7 With the above notation, $f \omega$ is McShane integrable, then so is $|f| \omega$.

The proof begins with replacing $\omega$ by its total equivalence $d F$, where $F$ is a primitive of $\omega$, which is some continuous monotonely increasing function. The advantage of this is that $d F$ is an interval function: $d F(t, I)$ does not depend on $t$. After this step we use a "Cauchy criterion in McShane style" to prove this theorem. A rather surprising fact from this line of argument is 
THEOREM 1.8 If $\omega$ is a positive totalizable integrator of style $\mathcal{S}_{\max }$ and if $f$ is a Borel function on $[a, b]$ such that the set $\mathcal{S}(f \omega, \gamma)$ is bounded for some gauge $\gamma$, then $f \omega$ is McShane integrable.

In conversation, Dr.Fong points out that one motivation for the theoretical development in [Fo2] is to prove theorems of this nature in other interesting situations. One of them is Theorem 1.10 below.

From what we have so far the equivalence of the following conditions for a Borel function $f$ on $[a, b]$ is routine to establish: 1. $f \in L^{1}[a, b] ; 2 . f$ is McShane integrable, i.e. the integrator $f d x$ is totalizable in style $\mathcal{S}_{\max }, 3 . f$ is Kurzweil-Henstock integrable (i.e. $f d x$ is totalizable in style $\mathcal{S}_{\text {bd }}$ ) and its primitive $F$ (given by $F(x)=\int_{a}^{x} f$ ) is of bounded variation.

The most substantial and significant part in the work [Fo2], which is influenced by Saks [Sak], deals with local variations of integrators. In this part of work, it is necessary to avoid "wild" integrators such as $d F$, where $F$ is a continuous but nowhere differentiable function. We say that an integrator $\alpha$ of style $\mathcal{S}$ is tame if there is a positive totalizable integrator $\omega$ such that

$$
\limsup _{I \rightarrow t,(t, I) \in \mathcal{S}} \frac{|\alpha(t, I)|}{\omega(t, I)}<+\infty .
$$

In this definition the style $\mathcal{S}$ is arbitrary, but in what follows we are only interested in the bound style $\mathcal{S}_{\mathrm{bd}}$. Here is the main theorem about tame integrators:

THEOREM 1.9 (decomposition of tame integrators) If $\alpha$ is a tame integrator of style $\mathcal{S}_{\mathrm{bd}}$, then $\alpha$ is totally equivalent to $f \mu-\beta$, where $\mu$ is a finite measure, $f$ is a Borel function and $\beta$ is a tame positive integrator with $\int_{-a}^{b} \beta=0$. If furthermore, $\alpha$ is totalizable, then $\beta=0$.

We give an example of "badly behaved" tame integrator $\beta: \beta(t, I)=|I|$ if the end points of $I$ are rational numbers and 0 otherwise. We can easily check that $0=\int_{-a}^{b} \beta<\bar{\int}_{a}^{b} \beta=1$. 
An application of this theorem is the following theorem, which is reminiscent of Perron's definition of integrals using majorization.

THEOREM 1.10 If $\omega$ is a totalizable atomless integrator of style $\mathcal{S}_{\mathrm{bd}}$, and $f$ is a Borel function such that $-\infty<\bar{\int}_{a}^{b} f \omega<+\infty$ and the function $F$ given by $F(x)=\bar{J}_{a}^{x} f \omega$ is continuous, then $f \omega$ is totalizable.

It is not clear if the continuity assumption on $F$ can be dropped.

We have seen the definition of functions of $\sigma$-bounded variation in the last section. We have

THEOREM 1.11 (Structural theorem for functions of $\sigma$-bounded variation) Let $F$ be a continuous function on $[a, b]$ with $F(a)=0$. Then the following conditions are equivalent:

1. F is of $\sigma$-bounded variation;

2. There is a tame totalizable integrator a of style $\mathcal{S}_{\mathrm{bd}}$ such that $F(x)=\int_{a}^{x}$ a for all $x \in[a, b]$;

3. There is a finite measure $\mu$ and a Borel function $f$ on $[a, b]$ such that $f d \mu$ is totalizable in style $\mathcal{S}_{\mathrm{bd}}$ and $F(x)=\int_{a}^{x} f d \mu$ for all $x \in[a, b]$.

Let $F$ be a continuous function on $[a, b]$ and $\mu$ be a finite positive Borel measure without atoms on $[a, b]$. We say that $F$ is absolutely continuous relative to a set $A$ in $[a, b]$ with respect to measure $\mu$ if, given $\varepsilon>0$, there exist $\delta>0$ such that whenever $\left\{\left[u_{j}, v_{j}\right]\right\}_{j \in J}$ is a finite set of nonoverlapping intervals in $[a, b]$ with end points in $A$ and $\sum_{j \in J} \mu\left(\left[u_{j}, v_{j}\right]\right)<\delta$, we have $\sum_{j \in J} O_{F}\left[u_{j}, v_{j}\right]<\varepsilon$. We say that $F$ is $\sigma$-absolutely continuous with respect to $\mu$ if therc are countably many sets $A_{n}$ such that $\cup_{n=1}^{\infty} A_{n}$ and $F$ is absolutely continuous with respect to $\mu$ relative to $A_{n}$ for all $n$. 
THEOREM 1.12 (structural theorem for $\sigma$-absolutely continuous functions) $A$ function $F$ on $[a, b]$ is $\sigma$-absolutely continuous with respect to a measure $\mu$ without atoms if and only if there is a Borel function $f$ on $[a, b]$ such that the integrator $f d \mu$ is totalizable in style $\mathcal{S}_{\mathrm{bd}}$ and $F(x)=\int_{a}^{x} f d \mu$.

The proofs of above four major theorems were carefully worked out in [Fo2] through a series of lemmas (a total of seventeen), some of them are of interest on their own.

Kurzweil's 1957 pioneering paper was mainly motivated by the basic existence and uniqueness in differentiation. There is an entire chapter in [Fo2] dealing with differential equations of the form $d y=\alpha_{y}$, where $\alpha_{y}$ is an integrator depending on a parameter $y$. 


\section{Chapter 2}

\section{Vector-valued Gauge Integrals}

The theory vector-valued measures is a well-developed branch of analysis, and there is no lack of monographs and conference proceedings devoted to this topic; see, e.g. [DiU]. The problems it suggests also stimulate other areas of research such as the Banach Space theory and probability theory, especially the problem concerning the Radon-Nikodym property, which links analysis, geometry and Banach space and martingale convergence. But the theory of vector-valued gauge integration is relatively unknown.

In the present chapter we give a preliminary report on this topic and suggest some problems for future research. Many results here are more or less folklore and references to these works are hard to find, or even nonexistent. The content of the present chapter is based on the original longer version of [Fo1], with three sections removed before its publication.

The Orlicz-Pettis theorem (see [Day; p. 60], [Die; Chapter IV] or [DiU; p. 22]) in Banach space theory says that every weakly subseries convergent series is strongly convergent. It is natural to ask if there is a continuous version of this theorem. The weakly subseries convergence for series is naturally replaced by Pettis integrability for functions. An appropriate substitute for norm convergence for series is, as shown by Fong [Fol], the Henstock-Kurzweil integrable functions on a closed interval with values in a Banach space, which is the main subject of study in the present chapter. 


\subsection{Notation and terminology}

The notation and the terminology used in the present chapter are standard. Throughout the chapter, we fix a closed interval $[a, b]$ and a Banach space $E$ with its dual denoted by $E^{*}$. We state some standard theorems about series and sequences in Banach spaces. For their proofs, we refer to Day [Day] and Diestel [Die]. Works in vector measures and integration of vector-valued functions are numerous; see Diestel and Uhr [DiU] and the reference there. Comparatively, papers about vector-valued Henstock-Kurzweil integrals on $[a, b]$ are few.

Let $\sum_{n=1}^{\infty} x_{n}$ be a series in a Banach space $E$. We say that $\sum_{n=1}^{\infty} x_{n}$ converges in norm (weakly, respectively) if there is some $x \in E$ such that

$$
\lim _{n \rightarrow \infty}\left\|\sum_{k=1}^{n} x_{k}-x\right\|=0 \quad\left(\lim _{n \rightarrow \infty} x^{*}\left(\sum_{k=1}^{\infty} x_{k}-x\right)=0 \forall x^{*} \in E^{*} \text { respectively }\right)
$$

We say that $\sum_{n=1}^{\infty} x_{n}$ is strongly unconditionally (weakly unconditionally respectively) if, for every permutation $\pi$ of $\mathrm{N}$ (the set of all positive integers), the series $\sum_{n=1}^{\infty} x_{\pi(n)}$ is convergent in norm (weakly, respectively), to a limit clearly independent of $\pi$ ). We say that $\sum_{n=1}^{\infty} x_{n}$ is absolutely convergent if $\sum_{n=1}^{\infty}\left\|x_{n}\right\|<\infty$. Clearly we have

$$
\begin{aligned}
\text { absolutely convergent } & \Rightarrow \text { strong unconditional convergence } \\
& \Rightarrow \text { weak unconditonal convergence }
\end{aligned}
$$

The celebrated theorem of Orlicz-Pettis says that a series is weakly unconditionally convergent if and only if it is strongly unconditionally convergent. Here we should mention another celebrated theorem in Banach space theory: Dvoresky-Rogers theorem, which says that for a Banach space $E$ every unconditionally convergent series is absolutely convergent if and only if $E$ is finite-dimensional.

Let $f$ be a function from the closed interval $[a, b]$ into a Banach space $E$. We say that $f$ is Pettis integrable if $f$ is measurable (see [DiU; p. 41]) and, for every Borel set $A$ in $[a, b]$, there exists an element $v_{A}$ in $E$ such that, for all $e^{*}$ in $E^{*}$, we have $e^{*} f \in L^{1}[a, b]$ and

$$
e^{*} v_{A}=\int_{A} e^{*} f(x) d x
$$


See [DiU; p. 52]. Here $v_{A}$, which is uniquely determined by $f$ and $A$, will be denoted by $\int_{A} f(x) d x$ or simply $\int_{A} f$. A consequence of the Orlicz-Pettis theorem mentioned at the beginning is that, for a Pettis integrable function $f:[a, b] \rightarrow E$, the map $\mu$ from the Borel family of $[a, b]$ into $E$ defined by

$$
\mu(A)=\int_{A} f
$$

is a vector measure, that is, for a sequence $\left\{A_{n}\right\}$ of pairwise disjoint Borel sets in $[a, b]$, the series $\sum_{n=1}^{\infty} \mu\left(A_{n}\right)$ converges in norm to

$$
\mu\left(\bigcup_{n=1}^{\infty} A_{n}\right)
$$

and, as a consequence, the series $\sum_{n=1}^{\infty} \mu\left(A_{n}\right)$ is unconditionally convergent; see [DiU; Corollary 6 on p. 54].

Now we repeat the definition of Kurzweil-Henstock integrals from the previous chapter in this new setting. Given a function $f:[a, b] \rightarrow E$ and a tagged partition (or a tagged figure)

$$
(\mathcal{P}, T) \equiv \mathcal{P}^{T}=\left\{t_{j}, I_{j}\right\}_{j=1}^{N}
$$

the Riemann sum $S\left(f, \mathcal{P}^{T}\right)$ is the element in $E$ given by

$$
S\left(f, \mathcal{P}^{T}\right)=\sum_{j=1}^{N} f\left(t_{j}\right)\left|I_{j}\right|
$$

where $\left|I_{j}\right|$ stands for the length of the interval $I_{j}$. For each gauge $\gamma$ on $[a, b]$, we denote by $R(f, \gamma)$ the set of all Riemann sums $S\left(f, \mathcal{P}^{T}\right)$ with $\gamma$-fine tagged partitions $\mathcal{P}^{T}$ :

$$
R(f, \gamma)=\left\{S\left(f, \mathcal{P}^{T}\right) \mid \mathcal{P}^{T} \text { is a } \gamma \text {-fine tagged partition }\right\}
$$

For $A \subseteq[a, b]$, denote by $\Gamma A$ the set of all gauges on $A$. Denote by $\mathcal{H} \mathcal{K}(f)$ the collection of all sets of the form $R(f, \gamma)$ with $\gamma \in \Gamma[a, b]$ :

$$
\mathcal{H K}(f)=\{R(f, \gamma) \mid \gamma \in \Gamma[a, b]\} .
$$

Then $\mathcal{H} \mathcal{K}(f)$ is a net in $E$ :

$$
\gamma_{1} \geq \gamma_{2} \Rightarrow R\left(f, \gamma_{1}\right) \supseteq R\left(f, \gamma_{2}\right)
$$


We say that $f$ is Kurzweil-Henstock integrable, or simply HK-integrable, if this net is convergent, that is, for every $\varepsilon>0$, there exists some $\gamma \in \Gamma[a, b]$ such that diam $R(f, \gamma)<\varepsilon$. (Here we denote by diam $S$ the diameter of a nonempty set $S$ in $E$ :

$$
\operatorname{diam} S=\sup _{u, v \in S}\|u-v\|
$$

Thus, $f$ is HK-integrable if there is a (unique) element, $e$ in $E$ such that, given $\varepsilon>0$, there is $\gamma \in \Gamma[a, b]$ such that the set $R(f, \gamma)$ is within $\varepsilon$ of $e$, that is,

$$
R(f, \gamma) \subseteq B(e, \varepsilon):=\{x \in E \mid\|x-e\|<\varepsilon\}
$$

We call this unique element $e$ the Kurzweil-Henstock integral of $f$ and denote it by $\int_{a}^{b} f(x) d x$ or simply by $\int_{a}^{b} f$. When $E$ is $\mathbf{R}$ or $\mathbf{C}$, the definition of HK integrals here agrees with the usual one given in the previous chapter.

\subsection{Basic Properties of Vector-Valued Kurzweil-Henstock Integrals}

Many properties of scalar-valued HK-integrable functions can be easily extended to the vector-valued case. Here we state some of them which are either very basic or relevant to our later work. The proof of these properties will be brief since they are slight modifications of those for the scalar-valued case, which are well-known.

Proposition 2.1 (a) If a function $f:[a, b] \rightarrow E$ is HK-integrable, then its restriction to each subinterval of $[a, b]$ is also HK-integrable. If, furthermore, the union $J$ of a finite set $\left\{J_{k}\right\}_{k=1}^{n}$ of nonoverlapping intervals is an interval contained in $[a, b]$, then

$$
\int_{J} f=\sum_{k=1}^{n} \int_{J_{k}} f
$$

(b) If $f$ and $g$ are $H K$-integrable functions from $[a, b]$ into $E$, then so is $\alpha f+\beta g$ for arbitrary scalars $\alpha$ and $\beta$, and

$$
\int(\alpha f+\beta g)=\alpha \int f+\beta \int g \text {. }
$$


(c) If $T$ is a bounded linear map from $E$ into another Banach space $F$ and if $f:[a, b] \rightarrow E$ is HK-integrable, then $T f:[a, b] \rightarrow F$ is also HK-integrable and

$$
\int_{a}^{b} T f=T \int_{a}^{b} f
$$

Proof: For (a), it is enough to show that, if $a<c<b$, then $f$ is integrable over both $[a, c]$ and $[c, b]$, and $\int_{a}^{b} f=\int_{a}^{c} f+\int_{c}^{b} f$. This follows immediately from the obvious relation

$$
R(f, \gamma) \supseteq R\left(\left.f\right|_{[a, c]},\left.\gamma\right|_{[a, c]}\right)+R\left(\left.f\right|_{[c, b]},\left.\gamma\right|_{[c, b]}\right) \quad \forall \gamma \in \Gamma[a, b] .
$$

(b) follows from the obvious relation

$$
R\left(\alpha f+\beta g, \gamma_{1} \wedge \gamma_{2}\right) \subseteq \alpha R\left(f, \gamma_{1}\right)+\beta R\left(g, \gamma_{2}\right)
$$

for all $\gamma_{1}, \gamma_{2} \in \Gamma[a, b]$, where $\gamma_{1} \wedge \gamma_{2}$ is the gauge defined by

$$
\left(\gamma_{1} \wedge \gamma_{2}\right)(t)=\min \left\{\gamma_{1}(t), \gamma_{2}(t)\right\}
$$

(c) follows from

$$
R(T f, \gamma)=T(R(f, \gamma))
$$

and the continuity of $T . \diamond$

In the last chapter we have seen a technical result which plays an important role in HKintegration, the so-called Saks-Henstock's lemma. It is straightforward to generalize this result for vector-valued functions:

Proposition 2.2 Let $f:[a, b] \rightarrow E$ be HK-integrable and $A$ be an arbitrary set contained in $[a, b]$. Then, given $\varepsilon>0$, there exists a gauge $\gamma$ on $A$ such that

$$
\left\|\sum_{j=1}^{N}\left\{f\left(t_{j}\right)\left|I_{j}\right|-\int_{I_{j}} f\right\}\right\|<\varepsilon .
$$

for each $\gamma$-fine tagged figure $\left\{t_{j}, I_{j}\right\}_{j=1}^{N}$. 
Proof. By assumption, there exists a gauge $\delta$ on $[a, b]$ such that

$$
\left\|S\left(f, \mathcal{P}^{T}\right)-\int_{[a, b]} f\right\|<\varepsilon / 2
$$

for every $\delta$-fine tagged figure $(\mathcal{P}, T) \equiv \mathcal{P}^{T}$. Let $\gamma=\delta \mid A$ and let $\left(\mathcal{P}_{0}, T_{0}\right)=\left\{t_{j}, I_{j}\right\}_{j=1}^{N}$ be a $\gamma$-fine partial tagged-partition. Let $\left\{J_{k}\right\}_{k=1}^{M}$ be a collection of closed subintervals of $[a, b]$ such that, $\left\{I_{j}\right\}_{j}$ and $\left\{J_{k}\right\}_{k}$ together form a partition of $[a, b]$. By part (a) of the previous proposition, we see that $\left.f\right|_{J_{k}}$ is integrable for each $k$. Thus there exists a $\left.\delta\right|_{J_{k}}$-fine tagged partition $\left(\mathcal{P}_{k}, T_{k}\right)$ of $J_{k}$ such that

$$
\left\|S\left(\left.f\right|_{J_{k}}, \mathcal{P}_{k}^{T_{k}}\right)-\int_{J_{k}} f\right\|<\varepsilon / 2 M .
$$

Putting $\mathcal{P}=\bigcup_{k=0}^{M} \mathcal{P}_{k}$ and $T=\bigcup_{k=0}^{M} T_{k}$ we obtain a $\delta$-fine tagged partition $(\mathcal{P}, T)$ of $[a, b]$ so that (2.1) holds. Now the conclusion is clear from (2.1) and (2.2).

Corollary 2.3 The indefinite integral $F$ of a HK-integrable function $f:[a, b] \rightarrow E$ given by

$$
F(x)=\int_{a}^{x} f \quad(a \leq x \leq b)
$$

is norm-continuous.

Proof. To show that $F$ is continuous at a point $c$ in $[a, b]$, apply the above proposition to the singleton set $A=\{c\} . \diamond$

One intriguing problem related to the above proposition is: under what condition does a HK-integrable function $f:[a, b] \rightarrow E$ have the Saks-Henstock property? We say that $f:[a, b] \rightarrow E$ has the Saks-Henstock property if it is HK-integrable and if, given $\varepsilon>0$, there exists a gauge $\gamma$ on $[a, b]$ such that, for each $\gamma$-fine tagged partition $\left\{t_{j}, I_{j}\right\}_{j=1}^{N}$ of $[a, b]$, we have

$$
\sum_{j=1}^{N}\left\|f\left(t_{j}\right)\left|I_{j}\right|-\int_{I_{j}} f\right\|<\varepsilon
$$


It is easy to derive from Proposition 2 that a Kurzweil-Henstock integrable function on $[a, b]$ taking values in a finite dimensional space has the Saks-Henstock property. We shall see that Bochner integrable functions [DiU] on $[a, b]$ have the Saks-Henstock property. The main theorem in the next section will indicate why the Saks-Henstock property is interesting. A thorough answer to this "intriguing problem" here seems to be related to the geometry of the Banach space $E$, but at present we still have no clue.

From the previous corollary we see that if $f:[a, b] \rightarrow E$ is HK-integrable, then the restriction $\left.f\right|_{[a, c]}$ is HK-integrable for every $c \in(a, b)$ and the $\operatorname{limit} \lim _{c \nmid b} \int_{a}^{c} f$ exists. As mentioned in the last chapter, one of the most interesting results in Kurzweil-Henstock theory of integration is the converse of the above statement, called Hake's theorem. A consequence of this theorem is that improper integrals can be interpreted as HK-integrals. This result can be generalized for the vector-valued case, which is stated in part (b) of the following proposition.

Proposition 2.4 (a) A function $f:[a, b] \rightarrow E$ is $H K$-integrable if there is some $c \in[a, b]$ such that $\left.f\right|_{[a, c]}$ and $\left.f\right|_{[a, b]}$ are HK-integrable. (b) A function $f:[a, b] \rightarrow E$ is HKintegrable if there is a strictly increasing sequence $\left\{b_{n}\right\}$ in $[a, b]$ with $\lim _{n} b_{n}=b$ such that $\left.f\right|_{\left[a, b_{n}\right]}$ are HK-integrable for all $n$ and the norm-limit $\lim _{n} \int_{a}^{b_{n}} f$ exists.

We omit the proof of this proposition since the usual argument for the real-valued case works in the same way here.

Take a sequence $\left\{e_{n}\right\}_{n=1}^{\infty}$ in $E$ and a strictly increasing sequence $\left\{b_{n}\right\}_{n=0}^{\infty}$ such that $b_{0}=a$ and $b_{n} \uparrow b$. Define $f:[a, b] \rightarrow E$ by putting

$$
f(x)=\left(b_{n}-b_{n-1}\right)^{-1} e_{n} \quad \text { for } x \in\left[b_{n-1}, b_{n}\right) \quad(n=1,2, \ldots)
$$

and $f(b)=0$. Then we can check the following assertions:

1. the series $\sum_{n} e_{n}$ is absolutely convergent (that is, $\sum_{n=1}^{\infty}\left\|e_{n}\right\|<\infty$ ) if and only if $\int_{a}^{b} f$ is Bochner integrable; 
2. the series $\sum_{n} e_{n}$ is weakly unconditionally Cauchy (that is, $\sum_{n}\left|e^{*} e_{n}\right|<\infty$ for all $e^{*}$ in the dual $E^{*}$; see [Die; p. 44]) if and only if $e^{*} f \in L^{1}[a, b]$ for all $e^{*} \in E^{*}$; and

3. the series $\sum_{n} e_{n}$ is norm-convergent if and only if $f:[a, b] \rightarrow E$ is Kurzweil-Henstock integrable; (this follows from the above proposition.)

These assertions motivate the major work in the following sections.

\subsection{Bochner Integrals Are Kurzweil-Henstock Integrals}

Since the HK-integrability of a function $f:[a, b] \rightarrow E$ means the convergence of the net $\mathcal{H} \mathcal{K}(f) \equiv\{R(f, \gamma)\}_{\gamma \in \Gamma[a, b]}$, it is of interest to find some "control" over $R(f, \gamma)$. This consideration leads to the following:

THEOREM 2.5 Suppose that $e_{0}$ be a fixed element in the Banach space $E,[a, b]$ is the union of countably many mutually disjoint sets $A_{n}(n=1,2, \ldots)$ and $\left\{r_{n}\right\}_{n=1}^{\infty}$ is a sequence of positive numbers such that $\left\|f(x)-e_{0}\right\| \leq r_{n}$ for all $x \in A_{n}$. Then, for each $\varepsilon>0$, there is a gauge $\gamma$ on $[a, b]$ such that $R(f, \gamma)$ is contained in a ball centered at $\frac{1}{b-a} e_{0}$ with radius

$$
R:=\varepsilon+\sum_{n=1}^{\infty} r_{n} \lambda^{*}\left(A_{n}\right)
$$

where $\lambda^{*}\left(A_{n}\right)$ is the Lebesgue outer measure of $A_{n}$.

Proof. If $R=\infty$, there is nothing to prove. So we assume $R<\infty$. By a slight change of notation, we may assume $e_{0}=0$. For each $n$, let $\mathcal{J}_{n}$ be a countable family of open sets covering $A_{n}$ such that

$$
\sum_{J \in \mathcal{J}_{n}}|J|<\lambda^{*}\left(A_{n}\right)+2^{-n_{n}} r_{n}^{-1} \varepsilon
$$

For each $t \in A_{n}$, let $\gamma(t)>0$ be such that the interval $(t-\gamma(t), t+\gamma(t))$ is contained in some open interval $J$ belonging to the collection $\mathcal{J}_{n}$. This defines a gauge $\gamma$ on $[a, b]$. 
Let $(\mathcal{P}, T)=\left\{t_{j}, I_{j}\right\}_{j=1}^{N}$ be a $\gamma$-fine tagged partition of $[a, b]$. We are going to show that $\left\|S\left(f, \mathcal{P}^{T}\right)\right\| \leq R$. Putting $T_{n}=T \cap A_{n}$, we have

$$
\sum_{t_{j} \in T_{n}}\left|I_{j}\right|<\lambda^{*}\left(A_{n}\right)+2^{-n} r_{n}^{-1} \varepsilon
$$

due to the fact that the intervals $I_{j}$ with $t_{j} \in T_{n}$ are nonoverlapping and each of them is contained in some interval from $\mathcal{J}_{n}$. So

$$
\left\|S\left(f, \mathcal{P}^{T}\right)\right\|=\left\|\sum_{n \geq 1} \sum_{s_{j} \in T_{n}} f\left(t_{j}\right)\left|I_{j}\right|\right\| \leq \sum_{n \geq 1} r_{n}\left(\lambda^{*}\left(A_{n}\right)+2^{-n} r_{n}^{-1} \varepsilon\right)=R .
$$

which is what we wanted to show.

Corollary 2.6 If $f:[a, b] \rightarrow E$ vanishes almost everywhere, then $f$ is HK-integrable with $\int_{a}^{b} f=0$ and $f$ has the Saks-Henstock property.

Proof. Let $A_{0}=\{x \in[a, b] \mid f(x)=0\}$ and

$$
A_{n}=\{x \in[a, b] \mid n-1 \leq\|f(x)\|<n\}
$$

for $n \geq 1$. Then $\lambda^{*}\left(A_{n}\right)=0$ for $n \geq 1$. By Theorem 2.5, given $\varepsilon>0$ there is a gauge $\gamma$ on $[a, b]$ such that $\left\|S\left(f, \mathcal{P}^{T}\right)\right\|<\varepsilon$ for all $\gamma$-fine tagged partition $\mathcal{P}^{T}$. So $f$ is HK-integrable and $\int_{a}^{b} f=0$. Since the function $\|f\|:[a, b] \rightarrow \mathbf{R}$ vanishes almost everywhere, it is also HK-integrable and $\int_{a}^{b}\|f\|=0$. Thus, given $\varepsilon>0$, there is a gauge $\gamma$ on $[a, b]$ such that for every $\gamma$-fine tagged partition $\left\{t_{j}, I_{j}\right\}_{j=1}^{N}$, we have, by noticing that $\int_{a}^{b}\|f\|=0$ and $\int_{a}^{b} f=0$,

$$
\sum_{j=1}^{N}\left\|f\left(t_{j}\right)\left|I_{j}\right|-\int_{I_{j}} f\right\| \leq\left|\sum_{j=1}^{N}\left\|f\left(t_{j}\right)\right\|\right| I_{j}\left|-\int_{a}^{b}\|f\|\right|<\varepsilon
$$

Hence $f$ has the Saks-Henstock property. $\diamond$

Corollary 2.7 Bochner integrable functions on $[a, b]$ are Kurzweil-Henstock integrable and their integrals in two senses coincide. 
Proof. Let $f:[a, b] \rightarrow E$ be a Bochner integrable function. According to Diestel and Uhl[DiU, p. $44, \S I I .2])$, there is a sequence of simple functions $\left\{\varphi_{n}\right\}$ from $[a, b]$ to $E$ such that $\int_{a}^{b}\left\|\varphi_{n}-f\right\| \rightarrow 0$. Simple functions are clearly HK-integrable. So it is enough to show that if $\left\{f_{n}\right\}$ is a sequence of HK-integrable functions such that $\int_{a}^{b}\left\|f_{n}-f\right\| \rightarrow 0$, then $f$ is also HK-integrable. Let $\varepsilon>0$ be given. Take a large enough $N$ such that $\int_{a}^{b}\left\|f_{N}-f\right\|<\varepsilon$. By Proposition 2.2, it is easy to see that there is a gauge $\gamma_{1}$ on $[a, b]$ such that $R\left(f_{N}-f, \gamma_{1}\right)$ is within a radius of $2 \varepsilon$ from the origin of $E$. Next, take a gauge $\gamma_{2}$ of $[a, b]$ such that $\operatorname{diam} R\left(f_{N}, \gamma_{2}\right)<\varepsilon$. Let $\gamma=\min \left\{\gamma_{1}, \gamma_{2}\right\}$. Write (B) $\int_{a}^{b} f$ the integral of $f$ in the Bochner sense. Then, for a $\gamma$-fine tagged partition $\mathcal{P}^{T}, S\left(f, \mathcal{P}^{T}\right)-(\mathrm{B}) \int_{a}^{b} f$ can be rewritten as

$$
S\left(f-f_{N}, \mathcal{P}^{T}\right)+S\left(f_{N}, \mathcal{P}^{T}\right)-(\mathrm{B}) \int_{a}^{b} f_{N}-(\mathrm{B}) \int_{a}^{b}\left\{f-f_{N}\right\}
$$

and hence its norm is less than $4 \varepsilon$. Therefore $f$ is HK-integrable and its integral coincides with (B) $\int_{a}^{b} f$.

Later we will see a result much stronger than this corollary, namely, every Pettis integrable function on $[a, b]$ is HK-integrable.

\subsection{Bochner Integrable Functions Have the Saks-Henstock Property}

Next we study the differential property of the indefinite integral $F$ of a Kurzweil-Henstock integrable function $f:[a, b] \rightarrow E$, given by $F(x)=\int_{a}^{x} f$ with $a \leq x \leq b$. For each $x \in(a, b)$, let

$$
\tilde{f}(x)=\lim \sup _{\delta, \eta \downarrow 0}\left\|\frac{F(x+\delta)-F(x-\eta)}{\delta+\eta}-f(x)\right\| \equiv \lim \sup _{\delta, \eta \downarrow 0}\left\|\frac{1}{\delta+\eta} \int_{x-\eta}^{x+\delta} f-f(x)\right\| .
$$

Clearly $F$ is differentiable at $x$ with $F^{\prime}(x)=f(x)$ if and only if $\tilde{f}(x)=0$. Now we show that, under the further assumption that $f$ has the Saks-Henstock property, $\tilde{f}=0$ almost 
everywhere. Take an arbitrary $\alpha>0$, let

$$
S_{\alpha}=\{x \in[a, b] \mid \tilde{f}(x)>\alpha\}
$$

and let $K$ be a compact subset of $S_{\alpha}$. It is enough to show that $K$ is negligible. Let $\varepsilon>0$ be given. Take a gauge $\gamma$ on $[a, b]$ having the property that

$$
\sum_{j=1}^{N}\left\|f\left(t_{j}\right)\left|I_{j}\right|-\int_{I_{j}} f\right\|<\varepsilon \text { for every } \gamma \text {-fine partition }\left\{t_{j}, I_{j}\right\}_{j=1}^{N} \text { of }[a, b] \text {. }
$$

For each $x \in S_{\alpha}$ there exist positive numbers $\delta(x)$ and $\eta(x)$, both less than $\gamma(x)$, such that

$$
\left\|\frac{1}{\delta(x)+\eta(x)} \int_{x-\eta(x)}^{x+\delta(x)} f-f(x)\right\|>\alpha .
$$

Putting $I_{x}=(x-\eta(x), x+\delta(x))$, the last inequality can be rewritten as

$$
\left\|\int_{I_{x}} f-f(x)\left|I_{x}\right|\right\|>\alpha\left|I_{x}\right|
$$

The collection $\left\{I_{x}\right\}_{x \in S_{\alpha}}$ covers $K$ and hence can be reduced to a finite subcover, say $\left\{I_{x}\right\}_{x \in F}$, where $F$ is a finite set in $K$. It follows from an elementary covering lemma of Vitali type ([Ste; $\S 1.6$ in Chapter 1]) that there are finitely many points $x_{k}(1 \leq k \leq n)$ in $F$ such that the intervals $J_{k}:=I_{x_{k}}$ are nonoverlapping and

$$
\lambda(K) \leq 5 \sum_{k=1}^{n}\left|J_{k}\right|
$$

(here $\lambda(K)$ stands for the Lebesgue measure of $K$.) The $\gamma$-fine tagged figure $\left\{J_{k}, x_{k}\right\}_{k=1}^{n}$ can be extended to a $\gamma$-fine tagged partition $\left\{t_{j}, I_{j}\right\}_{j=1}^{N}$ of $[a, b]$ so that (2.3) holds. Thus we have $\sum_{k}\left\|f\left(x_{k}\right)\left|J_{k}\right|-\int_{J_{k}} f\right\|<\varepsilon$. Hence

$$
\alpha \lambda(K) \leq 5 \sum_{k=1}^{n} \alpha\left|J_{k}\right| \leq 5 \sum_{k=1}^{n}\left\|f\left(x_{k}\right)\left|J_{k}\right|-\int_{J_{k}} f\right\|<\varepsilon .
$$

Thus $\lambda(K)=0$. We have proved: 
Proposition 2.8 If $f:[a, b] \rightarrow E$ is HK-integrable and has the Saks-Henstock property, then its indefinite integral $F(x)=\int_{a}^{x} f$ is differentiable with $F^{\prime}=f$ a.e.

Corollary 2.9 Let $f:[a, b] \rightarrow E$ be a Kurzweil-Henstock integrable function. Assume that either $E$ is separable or $f$ has the Saks-Henstock property. Then: (a) $f$ is measurable, and (b) $f$ is uniquely determined by its indefinite integral, that is, $\int_{a}^{x} f=0$ for all $x \in[a, b]$ implies $f=0$ a.e.

Proof. By the Pettis measurability theorem (see [Die; p. 25]), to show (a), it is enough to check that $f$ has an essentially separable range and $f$ is weakly measurable. Let $F(x)=\int_{a}^{x} f$ for $x \in[a, b]$. For each $e^{*} \in E^{*}, e^{*} f$ is HK-integrable and has the Henstock property. By the above proposition, we have

$$
e^{*} f(x)=\lim _{n \rightarrow \infty} n \int_{x}^{x+1 / n} e^{*} f=\lim _{n \rightarrow \infty} n\left(e^{*} F(x+1 / n)-e^{*} F(x)\right)
$$

for almost all $x$. Thus $e^{*} f$, being the pointwise limit of a sequence of continuous functions except on a negligible set, is measurable. Now assume $E$ separable. Then (a) is clear. Also, we can take a countable set $D$ separating points of $E$ such that (2.5) holds for all $e^{*} \in D$ for almost all $x$. Thus, if $F(x)=0$ for all $x$, then $f(x)=0$ for all those $x$ for which 2.5 holds for all $e^{*} \in D$, from which we deduce $f=0$ a.e. This proves (b) under the assumption that $E$ is separable. In case $f$ has the Saks-Henstock property, we can apply the above proposition to $f$ directly and prove (a) and (b) by a similar argument.

THEOREM 2.10 For a continuous function $F:[a, b] \rightarrow E$ satisfying $F(a)=0$, the following two conditions are equivalent:

(a) There is an HK-integrable function $f:[a, b] \rightarrow E$ with the Saks-Henstock property such that $F(x)=\int_{a}^{x} f$ for all $x \in[a, b]$, 
(b) The derivative of $F$ exists almost everywhere and, given $\varepsilon>0$ and given a negligible set $A$, there is a gauge $\delta$ on $A$ such that

$$
\sum_{j=1}^{N}\left\|F\left(y_{j}\right)-F\left(x_{j}\right)\right\|<\varepsilon
$$

for every $\delta$-fine partial tagged-partition $\left\{t_{j},\left[x_{j}, y_{j}\right]\right\}_{j=1}^{N}$.

Proof. Assume condition (a). By the previous proposition, we know that the derivative of $F$ exists a.e. Let $A$ be any negligible set in $[a, b]$. Define a function $g$ on $[a, b]$ by putting $g(x)=-f(x)$ for $x \in A$ and $g(x)=0$ elsewhere. Then $g=0$ a.e. and hence $g$ is HK-integrable with $\int_{a}^{b} g=0$ and $g$ has the Saks-Henstock property. Let $h=f+g$. Then $h$ is also HK-integrable and $h$ also the Saks-Henstock property. (It is easy to check that the sum of two functions having the Saks-Henstock property has the same property.) Thus, given $\varepsilon>0$, there is a gauge $\delta$ on $A$ such that, for each $\delta$-fine tagged figure $\left\{t_{j}, I_{j}\right\}_{j=1}^{N}$, we have

$$
\sum_{j=1}^{N}\left\|h\left(t_{j}\right)\left|I_{j}\right|-\int_{I_{j}} h\right\|<\varepsilon
$$

But $h\left(t_{j}\right)=0$ when $t_{j} \in A$ and $\int_{I_{j}} h=\int_{I_{j}} f$, since $g=0$ a.e. Now (b) is clear.

Next we assume (b). There is a negligible set $A$ in $[a, b]$ such that the derivative $F^{\prime}(t)$ exists for all $t \in[a, b] \backslash A$. With no loss of generality, we assume $f$ vanishing on $A$. Let $\varepsilon>0$ be given. Then there is a gauge $\delta$ on $A$ satisfying the condition described in (b). For every $t \in[a, b] \backslash A$, there is a positive number $\delta_{t}$ such that

$$
\|F(x)-F(t)-f(t)(x-t)\|<\varepsilon|x-t|
$$

for all $x \in\left(t-\delta_{t}, t+\delta_{t}\right)$. It is easy to verify that, for all $x, y \in\left(t-\delta_{t}, t+\delta_{t}\right)$ with $x<t<y$,

$$
\|F(y)-F(x)-f(t)(y-x)\|<\varepsilon(y-x) .
$$

Define a gauge $\gamma$ on $[a, b]$ by putting $\gamma(t)=\delta(t)$ for $t \in A$ and $\gamma(t)=\delta_{t}$ for $t \notin A$. Let $\mathcal{P}^{T}=\left\{t_{j}, I_{j}\right\}_{j=1}^{N}$ be a $\gamma$-fine partition of $[a, b]$. Then 


$$
\sum_{t_{j} \notin A}\left\|F\left(x_{j}\right)-F\left(x_{j-1}\right)-f\left(t_{j}\right)\left(x_{j}-x_{j-1}\right)\right\|<\sum_{t_{j} \notin A} \varepsilon\left(x_{j}-x_{j-1}\right) \leq \varepsilon(b-a),
$$

and $\sum_{t_{j} \in A}\left\|F\left(x_{j}\right)-F\left(x_{j-1}\right)\right\|<\varepsilon$. Since $f\left(t_{j}\right)=0$ for $t_{j} \in A$, we can combine the last two inequalities into one

$$
\sum_{j=1}^{N}\left\|F\left(x_{j}\right)-F\left(x_{j-1}\right)-f\left(l_{j}\right)\left(x_{j}-x_{j-1}\right)\right\|<\varepsilon(b-a+1) .
$$

Thus

$$
\left\|F(b)-S\left(f, \mathcal{P}^{T}\right)\right\|=\left\|\sum_{j=1}^{N}\left[F\left(x_{j}\right)-F\left(x_{j-1}\right)\right]-\sum_{j=1}^{N} f\left(t_{j}\right)\left(x_{j}-x_{j-1}\right)\right\|
$$

is less than $\varepsilon(b-a+1)$.

This shows that $f$ is HK-integrable with $\int_{a}^{b} f=F(b)$. The same argument shows that $\int_{a}^{x} f=F(x)$ for all $x \in[a, b]$. Thus (2.6) tells us that $f$ has the Saks-Henstock property. $\diamond$.

Corollary 2.11 Bochner integrable functions have the Saks-Henstock property.

Proof. It is known that the indefinite integral $F(x)=\int_{a}^{x} f$ of a Bochner integrable function $f$ is differentiable at $x$ with $F^{\prime}(x)=f(x)$ for almost all $x$; ([DiU; p. 49, Theorem 9]. Thus the first part of condition (b) in the above proposition is satisfied. The second part follows from the absolute continuity of the function $F$; [DiU; p. 46, Theorem 4 (i)]. Now the corollary is clear.

\subsection{Pettis Integrable Functions are Kurzweil-Henstock Integrable}

The present section reproduces the main result of Fong [Fol]: Pettis integrable functions on $[a, b]$ into $E$ are Kurzweil-Henstock integrable. As the title of that paper suggested, which 
we have mentioned in the introduction, this theorem can be considered as the continuous version of the celebrated theorem of Orlicz-Pettis in Banach space theory, namely, every weakly subseries convergent series in any Banach space is strongly subseries convergent. We speculate that when Pettis introduced what is now called Pettis integrals, he had a continuous version of this subseries convergent theorem in mind to work on, but he was unable to substitute the strong subseries convergence by an appropriate type of vectorvalued integrals, identified as the vector-valued Kurzweil-Henstock theorem, which only appeared quite recently.

In order to make our argument run smoothly, first we state some known facts about Pettis integrals, which are largely taken from Diestel and Uhl [DiU], so that we can use them freely in our proof.

Let $f:[a, b] \rightarrow E$ be a Pettis integrable function and denote by $F$ its indefinite integral: $F(x)=\int_{a}^{x} f$ with $a \leq x \leq b$. Recall that the Pettis integrability for $f$ means that $f$ is measurable and, for each Borel set $A$ in $[a, b]$, there is an element $v_{A}$ in $E$ such that $e^{*} f \in L^{1}[a, b]$ and $e^{*} v_{A}=\int_{A} e^{*} f$ for all $e^{*} \in E^{*}$. We denote this element $v_{A}$ by $\int_{A} f$ and, in case $A$ is an interval, say $A=[c, d]$, we also denote it by $\int_{c}^{d} f$. If follows immediately from the Orlicz-Pettis theorem that $\mu(A)=\int_{A} f$ defines a vector-valued measure $\mu$ : for every sequence $\left\{A_{n}\right\}_{n=1}^{\infty}$ of mutually disjoint Borel sets, the series $\sum_{n=1}^{\infty} \mu\left(A_{n}\right)$ converges in norm to $\mu\left(\cup_{n=1}^{\infty} A_{n}\right)$; see [DiU; p. 53, Theorem 5]. Hence $\mu$ is bounded in sense that there is some $M>0$ such that $\|\mu(A)\| \leq M$ for all Borel sets $A$ in $[a, b]$; see [DiU]; p. 9, Corollary 19]. It follows that, for every $e^{*} \in E^{*}$, the measure $e^{*} \mu$ is of bounded variation and its total variation $\left\|e^{*} \mu\right\|$ does not exceed $4 M\left\|e^{*}\right\|$; see [DiU; p. 4, Proposition 11]. Notice that the total variation $\left\|e^{*} \mu\right\|$ here is just the total variation $V_{a}^{b}\left(e^{*} F\right)$ of $e^{*} F$ here, which is defined by

$$
V_{a}^{b}\left(e^{*} F\right)=\sup \sum_{k=1}^{N}\left|e^{*} F\left(x_{k}\right)-e^{*} F\left(x_{k-1}\right)\right|
$$

where the supremum is taken over all partitions $\mathcal{P}=\left\{\left[x_{k-1}, x_{k}\right]\right\}_{k=1}^{N}$ of $[a, b]$. Thus, for every partition $\left\{\left[x_{k-1}, x_{k}\right]\right\}_{k=1}^{N}$ of $[a, b]$ and for each $e^{*} \in E^{*}$ with $\left\|e^{*}\right\| \leq 1$, we have 


$$
\sum_{k=1}^{N}\left|e^{*} F\left(x_{k}\right)-e^{*} F\left(x_{k-1}\right)\right| \leq 4 M
$$

Clearly $\mu(A)=0$ for each Borel set $A$ with zero Lebesgue measure. It follows that, for every $\varepsilon>0$, there exists some $\delta>0$ such that $\|\mu(A)\|<\varepsilon$ for every Borel set $A$ with $\lambda(A)<\delta$; see [DiU, p. 10, Theorem 1 (Pettis)]. With no loss of generality, we shall assume $f$ is Borel measurable.

Let $A_{0}=\{x \in[a, b] \mid f(x)=0\}$ and

$$
A_{n}=\{x \in[a, b] \mid n-1<\|f(x)\| \leq n\}
$$

for positive integers $n$. Then $A_{n}(n \geq 0)$ are mutually disjoint and $[a, b]=\bigcup_{n=0}^{\infty} A_{n}$. Hence the series

$$
\sum_{n=0}^{\infty} \int_{A_{n}} f \equiv \sum_{n=0}^{\infty} \int_{a}^{b} \chi_{A_{n}} f
$$

converges (unconditionally) to $\int_{a}^{b} f$; here $\chi_{A_{n}}$ is the characteristic function of $A_{n}$. Note that unconditional convergence of a series in a Banach space, $\sum_{n=1}^{\infty} a_{n}<\infty$ is equivalent to the unordered convergence of this series: given $\varepsilon>0$, there exists a positive integer $N$ such that, for each finite set $J$ of positive integers which are $\geq N$, we have $\left\|\sum_{j \in J} a_{j}\right\|<\varepsilon$; [Day; p. 58].

Now let $\varepsilon>0$ be given. Then there exists $\delta>0$ such that $\|\mu(A)\|<\varepsilon$ for all Borel sets $A$ in $[a, b]$ with $\lambda(A)<\delta$. Take a large enough positive integer $n_{0}$ such that

$$
\sum_{n>n_{0}} \lambda\left(A_{n}\right) \equiv \lambda\left(\bigcup_{n>n_{0}} A_{n}\right)<\delta
$$

For each positive integer $n$, since $\chi_{A_{n}} f$ is bounded and measurable, by [DiU, p. 45, Theorem 2], it is Bochner integrable. Hence, by Corollary 2.7 and Corollary 2.11 above, it: is also Henstock-Kurzweil integrable and it has the Saks-Henstock property. Thus there is a gauge $\gamma_{n}$ on $[a, b]$ such that, for every $\gamma_{n}$-fine tagged partition $\left\{t_{j}, I_{j}\right\}_{j=1}^{N}$ of $[a, b]$, we have 


$$
\sum_{j=1}^{N}\left\|\chi_{A_{n}}\left(t_{j}\right) f\left(t_{j}\right)\left|I_{j}\right|-\int_{Y_{j}} \chi_{A_{n}} f\right\|<\varepsilon / 2^{n}
$$

which also gives

$$
\left\|\sum_{j=1}^{N} \chi_{A_{n}}\left(t_{j}\right) f\left(t_{j}\right)\left|I_{j}\right|-\int_{a}^{b} \chi_{A_{n}} f\right\|<\varepsilon / 2^{n}
$$

Notice that (2.7) is trivial when $n=0$. For convenience, set $\gamma_{0}(t) \equiv 1$. Define a gauge $\gamma$ on $[a, b]$ by putting

$$
\gamma(t)= \begin{cases}\min \left\{\gamma_{0}(t), \gamma_{1}(t), \ldots, \gamma_{n_{0}}(t)\right\} & \text { if } t \in A_{n} \text { for some } n \leq n_{0} \\ \min \left\{\gamma_{0}(t), \gamma_{1}(t), \ldots, \gamma_{n}(t)\right\} & \text { if } t \in A_{n} \text { for some } n>n_{0}\end{cases}
$$

Let $\mathcal{P}^{T}=\left\{t_{j}, I_{j}\right\}_{j=1}^{N}$ be a $\gamma$-fine tagged partition of $[a, b]$. Let

$$
S_{n}=\left\{j \mid t_{j} \in A_{n}\right\}
$$

for $n \geq 0$. Then

$$
\begin{aligned}
& \left\|\sum_{j=1}^{N} f\left(t_{j}\right)\left|I_{j}\right|-\int_{a}^{b} f\right\| \\
& \quad=\left\|\sum_{n=0}^{n_{0}}\left\{\sum_{j \in S_{n}} f\left(t_{j}\right)\left|I_{j}\right|-\int_{A_{n}} f\right\}+\sum_{n>n_{0}} \sum_{j \in S_{n}} f\left(t_{j}\right)\left|I_{j}\right|-\int_{B_{0}} f\right\|,
\end{aligned}
$$

where $B_{0}=\bigcup_{n>n_{0}} A_{n}$. For $n \leq n_{0}$, the tagged partition $\mathcal{P}^{T}$ is $\gamma_{n}$-fine (because $\gamma(t) \leq \gamma_{n}(t)$ for all $t$ ) and hence

$$
\left\|\sum_{j \in S_{n}} f\left(t_{j}\right)\left|I_{j}\right|-\int_{A_{n}} f\right\|=\left\|\sum_{j=1}^{N} \chi_{A_{n}}\left(t_{j}\right) f\left(t_{j}\right)\left|I_{j}\right|-\int_{a}^{b} \chi_{A_{n}} f\right\|<\varepsilon / 2^{n}
$$

in view of the inequality following (2.7). For $n>n_{0}$, since $\left\{t_{j}, I_{j}\right\}_{j \in S_{n}}$ is a $\gamma_{n}$-fine partial tagged-partition, it is not hard to derive from (2.8) that 


$$
\sum_{j \in S_{n}}\left\|\chi_{A_{n}}\left(t_{j}\right) f\left(t_{j}\right)\left|I_{j}\right|-\int_{I_{j}} \chi_{A_{n}} f\right\| \equiv \sum_{j \in S_{n}}\left\|f\left(t_{j}\right)\left|I_{j}\right|-\int_{I_{j} \cap A_{n}} f\right\|<\varepsilon / 2^{n}
$$

Notice that

$$
A_{0}:=\bigcup_{n>n_{0}} \bigcup_{j \in S_{n}} I_{j} \cap A_{n}
$$

is a subset of $B_{0} \equiv \bigcup_{n>n_{0}} A_{n}$ and hence its Lebesgue measure is less than $\delta$. Thus

$$
\left\|\mu\left(A_{0}\right)\right\|=\left\|\sum_{n>n_{0}} \sum_{j \in S_{n}} \int_{I_{j} \cap A_{n}} f\right\|<\varepsilon .
$$

It is clear from (2.9) and the last inequality that

$$
\left\|\sum_{n>n_{0}} \sum_{j \in S_{n}} f\left(t_{j}\right)\left|I_{j}\right|\right\|<2 \varepsilon .
$$

Since $\lambda\left(B_{0}\right)=\lambda\left(\bigcup_{n>n_{0}} A_{n}\right)<\delta$, we have $\left\|\int_{B_{0}} f\right\|<\varepsilon$. This inequality, together with (2.5), (2.8) and (2.10), gives

$$
\left\|\sum_{j=1}^{N} f\left(t_{j}\right)\left|I_{j}\right|-\int_{a}^{b} f\right\|<5 \varepsilon .
$$

Now we can conclude:

THEOREM 2.12 Pettis integrable functions are Kurzweil-Henstock integrable.

It is natural to ask if a Pettis integrable function has the Saks-Henstock property, or more generally if a HK-integrable function has the Saks-Henstock property. It would be of great interest if the answer turns out to be depending on the geometry of the Banach space $E$. But at present we do not have any evidence for such speculations. The investigation of the Saks-Henstock property seems to be an important direction for future work. 


\subsection{Remarks}

In this last section we make two technical remarks. The first is that a Bochner integrable function on $[a, b]$ has the Saks-Henstock property can be derived directly from Theorem 2.5 as follows.

Suppose that $f:[a, b] \rightarrow E$ is Bochner integrable. Let $\varepsilon>0$ be given. Then, as shown in Theorem 2.5, there is a step function $g:[a, b] \rightarrow E$ such that $\int_{a}^{b}\|f-g\|<\varepsilon$. By Proposition 5, we see that there is a gauge $\gamma_{1}$ on $[a, b]$ such that $R\left(\|f-g\|, \gamma_{1}\right)$ is set in $\mathrm{R}$ within a radius of $2 \varepsilon$ from 0 . Since a step function has the Saks-Henstock property, we may take a gauge $\gamma_{2}$ such that, for every $\gamma_{2}$-fine tagged partition $\left\{I_{j}, t_{j}\right\}_{j=1}^{N}$,

$$
\sum_{j=1}^{N}\left\|g\left(t_{j}\right)\left|I_{j}\right|-\int_{I_{j}} g\right\|<\varepsilon .
$$

Let $\gamma=\min \left\{\gamma_{1}, \gamma\right\}$ and let $\left\{t_{j}, I_{j}\right\}_{j=1}^{N}$ be a $\gamma$-fine partition. Then (2.11) and the following inequality hold:

$$
\sum_{j=1}^{N}\left\|f\left(t_{j}\right)-g\left(t_{j}\right)\right\|\left|I_{j}\right|<2 \varepsilon
$$

Notice that

$$
\sum_{j=1}^{N}\left\|\int_{I_{j}} f-\int_{I_{j}} g\right\| \leq \int_{a}^{b}\|f-g\|<\varepsilon .
$$

Putting inequalities (2.11), (2.12) and the last inequality together, it is easy to see that

$$
\sum_{j=1}^{N}\left\|f\left(t_{j}\right)\left|I_{j}\right|-\int_{I_{j}} f\right\|<4 \varepsilon
$$

Hence $f$ has the Saks-Henstock property. Our argument here can be easily adapted for showing a more general statement:

Proposition 2.13 If a measurable function $f:[a, b] \rightarrow E$ can be approximated in $L^{1-}$ norm by functions having the Saks-Henstock property, then $f$ also has the Saks-Henstock property.

The next technical remark concerns a characterization of Bochner integrable functions among Pettis integrable functions. Let $f:[a, b] \rightarrow E$ be a Bochner integrable function: 
$\int_{a}^{b}\|f\|<\infty$ and let $F(x)=\int_{a}^{x} f(a \leq x \leq b)$ be its indefinite integral. We have seen that $f$ has the Saks-Henstock property and we can easily show that $F$ is a function of bounded variation. Indeed, if $\left\{\left[x_{j-1}, x_{j}\right]\right\}_{j=1}^{N}$ is a partition of $[a, b]$, then

$$
\sum_{j=1}^{N}\left\|F\left(x_{j}\right)-F\left(x_{j-1}\right)\right\|=\sum_{j=1}^{N}\left\|\int_{x_{j-1}}^{x_{j}} f\right\| \leq \int_{a}^{b}\|f\|<\infty .
$$

We can prove that the converse is also true as follows.

Suppose that an HK-integrable function $f:[a, b] \rightarrow E$ has the Henstock property and its indefinite integral $F:[a, b] \rightarrow E$ is a function with a finite total variation $v$. We are going to show that $f$ is Bochner integrable. From Corollary 2.9, we know that $f$ is measurable and hence it is enough to show that $\|f\| \in L^{1}[a, b]$. Since $\|f\| \geq 0$, it is enough to show that $\|f\|$ is $\mathrm{HK}$-integrable. Here we have used the fact that an IIK-integrable realvalued function $\varphi$ on $[a, b]$ such that $\varphi \geq 0$ a.e. is also Lebesgue integrable. This can be seen as follows. Construct a sequence of bounded measurable functions $\varphi_{n} \geq 0$ such that $\varphi_{n}(x)$ converges to $\varphi(x)$ for almost all $x$ in $[a, b]$. Since the $\varphi_{n}$ 's are bounded, their integrals in the Lebesgue sense coincide with the Henstock-Kurzweil sense; see Corollary 2.7 above. Now use the fact that the monotone convergence theorem holds for both Lebesgue integrals and the HK-integrals.

Let $\varepsilon>0$ be given. Take a partition $\left\{\left[a_{k-1,1}, a_{k}\right]\right\}_{k=1}^{m}$ of $[a, b]$ such that

$$
v-\varepsilon<\sum_{k=1}^{m}\left\|F\left(a_{k}\right)-F\left(a_{k-1}\right)\right\| \leq v .
$$

By Corollary $3, F$ is norm continuous. Hence there exists $\delta>0$ such that, for all $k$ and all $x \in[a, b]$ with $\left|x-a_{k}\right|<\delta$, we have $\left\|F\left(a_{k}\right)-F(x)\right\|<\varepsilon / 2 m$. By the Saks-Henstock property of $f$, there is a gauge $\gamma$ on $[a, b]$ such that $\gamma(t)<\delta$ for all $t$ and, for each $\gamma$-fine tagged partition $\left\{\left[x_{j-1}, x_{j}\right], t_{j}\right\}_{j=1}^{N}$, we have

$$
\sum_{j=1}^{N}\left\|f\left(t_{j}\right)\left(x_{j}-x_{j-1}\right)-\int_{x_{j-1}}^{x_{j}} f\right\| \equiv \sum_{j=1}^{N}\left\|f\left(t_{j}\right)\left(x_{j}-x_{j-1}\right)-F\left(x_{j}\right)-F\left(x_{j-1}\right)\right\|<\varepsilon,
$$

from which it follows that 


$$
\left|\sum_{j=1}^{N}\left\|f\left(t_{j}\right)\right\|\left(x_{j}-x_{j-1}\right)-\sum_{j=1}^{N}\left\|F\left(x_{j}\right)-F\left(x_{j-1}\right)\right\|\right|<\varepsilon .
$$

Since $x_{j}-x_{j-1}<\delta$, it is easy to deduce from (2.13) and our choice of $\delta$ that

$$
v-2 \varepsilon<\sum_{j=1}^{N}\left\|F\left(x_{j}\right)-F\left(x_{j-1}\right)\right\| \leq v
$$

Thus it follows from (2.14) and (2.15) that

$$
\left|\sum_{j=1}^{N}\left\|f\left(t_{j}\right)\right\|\left(x_{j}-x_{j-1}\right)-v\right|<3 \varepsilon
$$

for every $\gamma$-fine tagged partition $\left\{t_{j},\left[x_{j-1}, x_{j}\right]\right\}_{j=1}^{N}$. We conclude:

Proposition 2.14 A Henstock-Kurzweil integrable function $f:[a, b] \rightarrow E$ is Bochner integrable if (a) it has the Saks-Henstock property: and (b) its indefinite integral $F$ is of bounded variation.

It seems that condition (a) in the above proposition can be dropped. But we are still unable to prove the proposition without this condition, perhaps because of our lack of understanding of the Saks-Henstock property at the present stage of research in vector-valued gauge integration. 


\section{Chapter 3}

\section{Riemann Type Product Integrals}

In this chapter we study multiplicative gauge integration of integrators with values in a topological group, or more generally, a topological monoid $G$. Published work in this area, according to Dr. E. Talvila (private communication), is rare. Here we mention Kurzweil and Jarnik [KuJ], which is a report on "Perron product integrals" of functions taking values in the general linear group $G L(n ; \mathbf{C})$. The proofs of their results were not included in that report and we do not know if their method works for more general situations that we are considering here.

We start this chapter with a rationale for studying this topic, which comes from Euler's constructive method of polygonal approximation in ordinary differential equations. Then we introduce some notation and terminolgy in topological groups and topological monoids to prepare for the study of multiplicative integration later in the chapter. After defining our gauge totalization of integrators with values in a topological group or more generally a topological monoid, and deriving its elementary properties, we prove a version of Hake's theorem in this setting. The proof is considerably more difficult than the original one since we cannot freely change the order of factors in a product. The discrepany which occurs in changing the order must be closely monitored and put under control.

In the final section, we prove an analogy of the known fact that a multiplicative measure is the exponential of an additive measure. This fact, although not deep, is used in continuous 
tensor products and Bose-Einstein quantum field theory; $[\mathrm{PaS}]$. We show that if $\omega$ is a totalizable multiplicative integrator on $[a, b]$ with values in the group of all invertible elements in a commutative Banach algebra $A$, then there is totalizable additive integrator $\alpha$ taking vector values in $A$ such that $\Pi_{I} \omega=\exp \int_{I} \alpha$ for all subintervals $I$ in $[a, b]$. We envisage that this result is still valid in some appropriate general setting, with the commutativity assumption of $A$ dropped.

\subsection{Preamble}

Before stating all relevant definitions for studying multiplicative gauge integration, we describe a well-known technique in differential equations for finding approximate trajectories to motivate this study, namely, Euler's method of polygons for constructing approximate solutions. Consider the initial value problem

$$
\frac{d y}{d t}=A(t) y ; \quad y\left(t_{0}\right)=y_{0}
$$

Here, $y_{0}$ is a fixed point in a Banach space (or more generally, a topological vector space) $Y, A(t)$ is an operator defined on $Y$, not necessarily linear, depending on a time parameter $t$ which varies in an open interval $I_{0}$, and $t_{0} \in I_{0}$ is a fixed time instant. Take any $T_{0} \in I_{0}$. For convenience, we assume $T_{0}>t_{0}$. Next, take a partition of $\left[t_{0}, T_{0}\right]$, say

$$
t_{0}<t_{1}<\ldots<t_{N}=T_{0}
$$

A polygonal curve in $\left[t_{0}, T_{0}\right] \times Y$ representing an approximate trajectory is obtained by a linear interpolation of the points

$$
\left(t_{0}, y_{0}\right),\left(t_{1}, y_{1}\right), \ldots,\left(t_{N}, y_{N}\right)
$$

which are defined inductively as follows. The first point $\left(t_{0}, y_{0}\right)$ is given by the initial condition $y\left(t_{0}\right)=y_{0}$. Assuming $\left(t_{k}, y_{k}\right)$ is known for some $k$ with $0 \leq k<N$, the point $\left(t_{k+1}, y_{k+1}\right)$ is determined by putting

$$
y_{k+1}=y_{k}+\left(t_{k+1}-t_{k}\right) A\left(t_{k}\right) y_{k} \equiv\left(1+\left(t_{k+1}-t_{k}\right) A\left(t_{k}\right)\right) y_{k}
$$


(Here, 1 stands for the identity operator.) Inductively, we get

$$
y_{N}=\left(1+\left(t_{N}-t_{N-1}\right) A\left(t_{N-1}\right)\right) \cdots\left(1+\left(t_{2}-t_{1}\right) A\left(t_{1}\right)\right)\left(1+\left(t_{1}-t_{0}\right) A\left(t_{0}\right)\right) y_{0}
$$

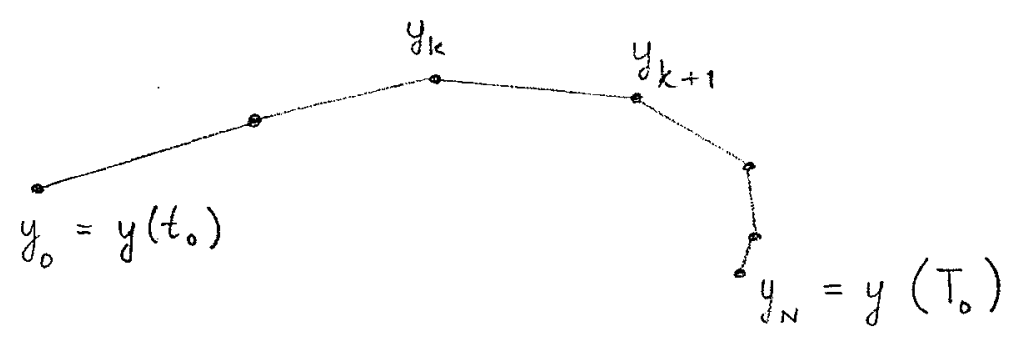

We can rewrite (3.2) as

$$
y_{N}=\omega\left(t_{N-1},\left[t_{N-1}, t_{N}\right]\right) \cdots \omega\left(t_{1},\left[t_{1}, t_{2}\right]\right) \omega\left(t_{0},\left[t_{1}, t_{0}\right]\right) y_{0}
$$

where $\omega$ is the operator-valued integrator defined by putting

$$
\omega(t, I)=1+(v-u) A(t)
$$

if $I=[u, v]$. By insisting a tag $t$ be the left endpoint of $I$ for evaluating $\omega(t, I)$ in (3.2) is imposing a restriction too stringent for developing a gauge integration. Instead we should begin with a tagged partition $P^{T}=\left\{\left(t_{k}, I_{k}\right)\right\}_{k=1}^{N}$ in any given style (with Cousin's Property!) of $\left[t_{0}, T_{0}\right]$ and consider the Riemann product

$$
\mathcal{P}\left(\omega, P^{T}\right)=\omega\left(t_{N}, I_{N}\right) \cdots \omega\left(t_{2}, I_{2}\right) \omega\left(t_{1}, I_{1}\right)
$$

where $t_{k}$ is not necessarily the left endpoint of $I_{k}$. Given a gauge $\gamma$ on $\left[t_{0}, T_{0}\right]$, denote by $\Pi(\omega ; \gamma)$ the set of all Riemann products $\mathcal{P}\left(\omega, P^{T}\right)$, where $P^{T}$ is any $\gamma$-fine tagged partition. We expect that, under some suitable condition, the nest (to be defined shortly), $\{\Pi(\omega, \gamma)\}_{\gamma}$ converges in some sense to an operator on $Y$ denoted by $F(T)$, which represents a product 
integral of the operator-valued integrator $\omega$. Also we expect that, in some favourable situations, $F(t)$ exists for all $t \in I_{0}$ and the map $t \in I_{0} \mapsto F(t) y_{0}$ gives a trajectory in $Y$ which solves the initial value problem (3.1).

To prepare the ground for the definitions of group-valued integrators, here we describe some basic terminology and notation about topological monoids and topological groups. By a monoid we mean a semigroup with an identity element. Recall that a semigroup is an algebraic system with a single binary operation which is associative. For simplicity in the future we call this binary operation multiplication. Thus, if $G$ is a semigroup with $G \times G \rightarrow G:(a, b) \mapsto a b$ as the binary operation, then $(a b) c=a(b c)$ for all $a, b, c \in G$. If, furthermore, 1 is an element in $G$ such that $a 1=1 a=a$ for all $a \in G$, then 1 is called an identity element of $G$. (It is easy to see that the identity element is unique, if it does exist.) An element $a$ in a monoid $G$ is said to be invertible if there exists an element $a^{-1}$ in $G$, which is uniquely determined by $a$ and is called the inverse of $a$, such that $a a^{-1}=a^{-1} a=1$. If every element in $G$ is invertible, then $G$ is a group.

Let $G$ be a monoid and suppose that $\mathcal{T}$ is a (Hausdorff) topology on $G$. We say that the pair $(G, \mathcal{T})$ is a topological monoid if the multiplication is continuous, in other words, the map $m: G \times G \rightarrow G$ defined by $m(a, b)=a b$ is continuous, where $G \times G$ is equipped with the product topology. Assuming that $(G, \mathcal{T})$ is a topological monoid with identity 1 , denote by $\mathcal{N}_{G}$ the neighbourhood system at 1 . Recall that a neighbourhood of 1 is a set $N$ in $G$ such that $1 \in V \subseteq N$ for some open set $V$ in $G$. The continuity of the map $m: G \times G \rightarrow G$ says that, for each $U \in \mathcal{N}_{G}$, there exists $V \in \mathcal{N}_{G}$ such that $V V \subseteq U$. (Here, $V V$ stands for the set $\{c \in G \mid c=a b$ for some $a, b \in V\}$. More generally, for $A, B \subseteq G$, we write $A B$ for the set $\{c \mid c=a b$ for some $a \in A$ and $b \in B\}$.) The elementary properties about the neighbourhood system $\mathcal{N}_{G}$ stated in the following lemmas (or rather, the arguments in establishing these lemmas) may be useful in the future:

Lemma 3.1 Given a topological monoid $G$ and $V \in \mathcal{N}_{G}$, there exists a sequence $\left\{V_{n}\right\}_{n \geq 1}$ in $\mathcal{N}_{G}$ such that $V_{1} V_{2} \cdots V_{n} \subseteq V$ and $V_{n} V_{n-1} \cdots V_{2} V_{1} \subseteq V$ for all $n$. 
Proof. We can define $V_{0}, V_{1}, V_{2}, \ldots$ inductively as follows. Let $V_{0}=V$. Once $V_{n} \in \mathcal{N}_{G}(n \geq 0)$ is obtained, we choose $V_{n+1} \in \mathcal{N}_{G}$ such that $V_{n+1} V_{n+1} \subseteq V_{n}$. Then

$$
\begin{aligned}
V=V_{0} & \supseteq V_{1} V_{1} \supseteq V_{1} V_{2} V_{2} \supseteq V_{1} V_{2} V_{3} V_{3} \supseteq \cdots \\
& \supseteq V_{1} V_{2} V_{3} \cdots V_{n-1} V_{n} V_{n} \supseteq V_{1} V_{2} \cdots V_{n-1} V_{n}
\end{aligned}
$$

Similarly, we can prove $V \supseteq V_{n} V_{n-1} \cdots V_{2} V_{1}$.

Lemma 3.2 Let $K_{1}$ and $K_{2}$ be compact sets in a topological monoid $G$ and let $W$ be an open set in $G$ such that $K_{1} K_{2} \subseteq W$. Then there exists $V \in \mathcal{N}_{G}$ such that $K_{1} V K_{2} \subseteq W$.

Proof. Assume on the contrary that for all $V \in \mathcal{N}_{G}, K_{1} V K_{2} \nsubseteq W$, that is, there exist $x_{V} \in K_{1}, y_{V} \in K_{2}$ and $a_{V} \in V$ such that $x_{V} a_{V} y_{V} \notin W$. Then $\left\{a_{V}\right\}_{V \in \mathcal{N}_{G}}$ is a net converging to 1 . Since $K_{1} \times K_{2}$ is compact, the net $\left\{\left(x_{V}, y_{V}\right)\right\}_{V \in \mathcal{N}_{G}}$ in $K_{1} \times K_{2}$ has a subnet, say $\left\{\left(x_{V(d)}, y_{V(d)}\right)\right\}_{d \in D}$, converging to to some $(x, y)$ in $K_{1} \times K_{2}$. Thus the net $\left\{x_{V(d)} a_{V(d)} y_{V(d)}\right\}_{d \in D}$ converges to $x y$. But $x_{V} a_{V} y_{V} \notin W$ for all $V \in \mathcal{N}_{G}$ and $W$ is open. So $x y \notin W$, contradicting $x y \in K_{1} K_{2} \subseteq W . \quad \diamond$

Lemma 3.3 Suppose that $G$ is a topological monoid and there is a compact set $K$ in $\mathcal{N}_{G}$. Then, given $W \in \mathcal{N}_{G}$, there is a sequence $\left\{V_{n}\right\}_{n \geq 1}$ of compact sets in $\mathcal{N}_{G}$ such that, for each positive integer $n$ and for each permutation $\sigma$ of $\{1, \ldots, n\}$, we have $V_{\sigma(1)} V_{\sigma(2)} \cdots V_{\sigma(n)} \subseteq W$

Proof. With no loss of generality, we assume that $W$ is open and precompact (i.e. it has a compact closure $\bar{W}$ ). We construct a sequence $\left\{V_{n}\right\}_{n \geq 1}$ in $\mathcal{N}_{G}$ inductively as follows. To construct $V_{1}$, first we notice that 1 is not in the compact set $\bar{W} \backslash W$ and hence there are disjoint open sets $U_{1}$ and $U_{2}$ such that $1 \in U_{1}$ and $\bar{W} \backslash W \subseteq U_{2}$. Let $V_{1}=\overline{U_{1} \cap W}$. Then $V_{1} \subseteq \bar{W}$ and hence $V_{1}$ is compact. Clearly $V_{1}$ does not intersect $U_{2}$ and hence $V_{1} \subseteq W$. Thus we have a compact set $V_{1}$ in $\mathcal{N}_{G}$ such that $V_{1} \subseteq W$. Next, suppose that $V_{1}, \ldots, V_{n}$ have been constructed. Then for each $\sigma \in S_{n}$ (as usual we write $S_{n}$ for the 
group of all permutations of $\{1, \ldots, n\})$ and each integer $k$ with $0 \leq k \leq n$, by applying the previous lemma to the cases

$$
K_{1}=V_{\sigma(0)} V_{\sigma(1)} \cdots V_{\sigma(k)} \quad \text { and } \quad K_{2}=V_{\sigma(k+1)} \cdots V_{\sigma(n)} V_{\sigma(n+1)}
$$

(here we adopt the convention that $V_{\sigma(0)}=V_{\sigma(n+1)}=\{1\}$ ), we can find some $W_{\sigma, k} \in \mathcal{N}_{G}$ such that

$$
V_{\sigma(0)} V_{\sigma(1)} \cdots V_{\sigma(k)} W_{\sigma, k} V_{\sigma(k+1)} \cdots V_{\sigma(n+1)} \subseteq W
$$

In particular $W_{\sigma, k} \subseteq W$, from which we see that $W_{\sigma, k}$ is precompact. Using the same argument as the one for constructing $V_{1}$, we can find a compact set $V_{\sigma, k} \in \mathcal{N}_{G}$ contained in $W_{\sigma, k}$. Let

$$
V_{n+1}=\bigcap_{\sigma \in S_{n}} \bigcap_{k=0}^{n} V_{\sigma, k} .
$$

It is easy to see that the sequence $\left\{V_{n}\right\}$ constructed in this way satisfies the requirement of this lemma.

For convenience, we will use the language of nests introduced at the beginning of [Fo2] to describe general types of convergence. A partially ordered set $D$ is said to be directed (downward) if, for all $d_{1}, d_{2} \in D$, there exists $d \in D$ such that $d \leq d_{1}$ and $d \leq d_{2}$. By a nest in a topological space $X$ we mean a collection of nonempty subsets indexed by a directed set $D$, say $\left\{A_{d}\right\}_{d \in D}$ such that $d_{1} \leq d_{2}$ implies $A_{d_{1}} \subseteq A_{d_{2}}$ for all $d_{1}, d_{2} \in D$. We say that the nest converges to a point $x_{0}$ if, for every neighbourhood $V$ of $x_{0}$, there exists some $d \in D$ such that $A_{d} \subseteq V$. If $X$ is a complete metric space, then a nest $\left\{A_{d}\right\}$ is convergent if and only if $\operatorname{diam} A_{d}$ shrinks to zero as $d$ decreases. Here we write diam $A_{d}$ of the diameter of $A_{d}$ ). If $X$ is the real line $\mathrm{R}$, then $\left\{A_{d}\right\}$ converges if and only if $-\infty<\underline{L}=\bar{L}<+\infty$, where $\bar{L}=\lim _{d} \sup A_{d}$ and $\underline{L}=\lim _{d} \inf A_{d}$. (The word "nest" is derived from the theorem about a sequence of nested intervals: if $\left\{I_{n}\right\}$ is a sequence of closed intervals such that $I_{n+1} \subseteq I_{n}$ for all $n$ and $\left|I_{n}\right| \rightarrow 0$ as $n \rightarrow \infty$, then $\bigcap_{n=1}^{\infty} I_{n}=\left\{x_{0}\right\}$ for some $x_{0} \in \mathbf{R}$.) 
In general a topological monoid is not naturally endowed with a uniform structure. In comparison, topological groups have a natural uniform structure and hence are more attractive for studying convergence. (recall that a topological group is a topological monoid $G$ such that all elements in $G$ are invertible and the inverse map $\iota: G \rightarrow G$ given by $\iota(a)=a^{-1}$ is continuous.) With a topological group $G$, we may ask if Cauchy's criterion is valid, which is a legitimate question due to its uniform structure. More precisely, a nest $\mathcal{N}_{\Lambda}=\left\{N_{\lambda}\right\}_{\lambda \in \Lambda}$ in a topological group $G$, with index set $\Lambda$ directed downward, is said to be a Cauchy nest if for every basic neighbourhood $U$ of 1 (that is, $U \in \mathcal{N}_{G}$ ) there is a $\lambda_{U} \in \Lambda$ such that, for all $\lambda<\lambda_{U} N_{\lambda} N_{\lambda}^{-1} \subseteq U$. A topological group $G$ is said to be complete if every Cauchy nest in $G$ is convergent. (The completeness of $G$ defined here is equivalent to the usual one which uses either filters, or nets in the sense of Moore and Smith.) Clearly, locally compact groups are complete.

Completeness for infinite dimensional groups requires more care. Let us only consider one example here: $\mathcal{U}_{H}$, the group of unitary operators on a separable, infinite dimmensional Hilbert space $H$. Unfortunately, in the weak operator topology under which $\mathcal{U}_{H}$ is not a Polish space: this group is not complete. It is complete under the norm topology, but unfortunately it is not even separable under the norm topology. This brings us to the consideration of the so-called $*$-strong topology, which is a well-known but seldom used topology in the theory of operator algebras. It is not hard to check that, under the $*$-strong topology, $\mathcal{U}_{H}$ is both a Polish space and a complete group.

\section{$3.2 \quad G$-valued Integrators}

Let $G$ be a topological monoid, and $[a, b]$ a closed bounded interval in $\mathbf{R}$, with $\mathcal{S}$ a style with Cousin's property on $[a, b]$. A $G$-valued integrator $\omega$ is a map $\mathcal{S} \longmapsto G$. For a $G$-valued integrator $\omega$ on $[a, b]$, and a tagged partition $P^{T}=\left\{\left(t_{k}, I_{k}\right)\right\}_{k=1}^{N}$ of $[a, b]$ in style $\mathcal{S}$, the Riemann Product for $\omega$, written as $\mathcal{P}\left(\omega, P^{T}\right)$ is given by

$$
\mathcal{P}\left(\omega, P^{T}\right)=\prod_{k=1}^{N} \omega\left(t_{N-k+1}, I_{N-k+1}\right) \equiv \omega\left(t_{N}, I_{N}\right) \omega\left(t_{N-1}, I_{N-1}\right) \cdots \omega\left(t_{1}, I_{1}\right) .
$$


The order in which the terms are multiplied is suggested by Euler's approximate trajectories for the initial value problem described in the last section. Given a gauge $\gamma$ on $[a, b]$ we define the sets

$$
\Pi(\omega, \gamma)=\left\{\mathcal{P}\left(\omega, P^{T}\right) \mid P^{T} \text { is } \gamma \text {-fine }\right\}
$$

and the nest

$$
\Pi(\omega)=\{\Pi(\omega, \gamma) \mid \gamma \text { is a gauge on }[a, b]\}=\{\Pi(\omega, \gamma)\}_{\gamma}
$$

We index the family $\Pi(\omega)$ by the set $\Gamma$ of all gauges on $[a, b]$, directed downward, to get a nest in $G$.

Definition 3.4 Let $\omega$ be a G-valued integrator on $[a, b]$. We say that $\omega$ is totalizable in style $\mathcal{S}$ if the nest $\Pi(\omega)$ converges. We call the limit of this nest the total and write $\prod_{a}^{b} \omega$ to denote this limit.

Our first example of a totalizable integrator is rather mundane. Let $X_{t}$ be a smooth, complete and time dependent vector field on a manifold $M$ and let $\Phi$ be the flow generated by $X_{t}$. (The completeness is guaranteed if the manifold is compact.) Let $G=\operatorname{Diff}(M)$, the group of all diffeomorphisms of $M$, equipped with the compact-open topology. Then, for all $u, v \in \mathbf{R}, \Phi(u, v)$ is an element in $G$. Furthermore, we have the following transition law:

$$
\Phi(u, u)=1, \quad \Phi(s, t) \Phi(t, u)=\Phi(s, u) .
$$

We define an integrator $\omega$ with values in $G$ by $\omega(t, I)=\Phi(u, v)$, where $I=[u, v]$. Then, for each tagged partition $P^{T}$ of $[a, b]$, we have $\Pi\left(\omega, P^{T}\right)=\Phi(b, a)$. Hence $\omega$ is totalizable with $\Pi_{a}^{b} \omega=\Phi(b, a)$.

The second example is more interesting. Let $G$ be a connected Lie group and let $I G$ be its Lie algebra. As usual we write $\exp : L G \rightarrow G$ for the exponential map. Let $\gamma:[a, b] \rightarrow L G$ be a curve in the linear space $L G$. We define a $G$-valued integrator $\omega$ by putting $\omega(t, I)=\exp (\gamma(v)-\gamma(u))$ if $t \in[a, b]$ and $I=[u, v]$. When $G$ is an abelian 
group, clearly $\omega$ is totalizable with $\Pi_{a}^{b} \omega=\exp (\gamma(b)-\gamma(a))$. When $G$ is nonabelian, the totalization of $\omega$ becomes a nontrivial problem which is beyond the scope of the present thesis. We anticipate that the Baker-Campbell-Hausdorff formula is needed to deal with this problem.

We now establish some elementary properties of totalization of $G$-valued integrators.

Proposition 3.5 Let $\omega$ be a $G$-valued integrator which is totalizable on $[a, b]$, where $G$ is a complete topological group. Then for any $c \in(a, b), \prod_{a}^{c} \omega$ and $\prod_{c}^{b} \omega$ exist, and $\left(\prod_{c}^{b} \omega\right)\left(\prod_{a}^{c} \omega\right)=\prod_{a}^{b} \omega$

Proof Let $U \in \mathcal{N}_{G}$ be a basic neighbourhood of 1 with $U^{-1}=U$. Take a gauge $\gamma$ on $[a, b]$ so that if $P^{T}$ and $Q^{S}$ are $\gamma$-fine tagged partitions of $[a, b]$ we have

$$
\mathcal{P}\left(\omega, P^{T}\right) \mathcal{P}\left(\omega, Q^{S}\right)^{-1} \in U
$$

(Equivalently we may write $\mathcal{P}\left(\omega, Q^{S}\right) \mathcal{P}\left(\omega, P^{T}\right)^{-1} \in U$ in view of $U=U^{-1}$.) Now take any two $\left.\gamma\right|_{[a, c]}$-fine tagged partitions $P_{0}^{T_{0}}=\left\{\left(t_{j}, I_{j}\right)\right\}_{j=1}^{N}$ and $P_{1}^{T_{1}}=\left\{\left(t_{k}^{\prime}, I_{k}^{\prime}\right)\right\}_{k=1}^{M}$ of $[a, c]$. By design, these partitions both include $c$ as the right endpoint of some tagged interval. Take any $\left.\gamma\right|_{[c, b]}$-fine tagged partition $R^{S}=\left\{\left(s_{i}, J_{i}\right)\right\}_{i=1}^{L}$ of $[c, b]$. Joining this to each of $P_{0}^{T_{0}}$ and $P_{1}^{T_{1}}$ gives two $\gamma$-fine tagged partitions of $[a, b]$, namely

$$
\begin{aligned}
P_{*}^{T_{*}} & =\left\{\left(t_{1}, I_{1}\right), \ldots,\left(t_{N}, I_{N}\right),\left(s_{1}, J_{1}\right), \ldots,\left(s_{L}, J_{L}\right)\right\} \\
\text { and } Q_{*}^{S_{*}} & =\left\{\left(t_{1}^{\prime}, I_{1}^{\prime}\right), \ldots,\left(t_{M}^{\prime}, I_{M}^{\prime}\right),\left(s_{1}, J_{1}\right), \ldots,\left(s_{L}, J_{L}\right)\right\}
\end{aligned}
$$

Since $c$ is the right endpoint of $I_{N}$ and $I_{M}^{\prime}$, and the left endpoint of $J_{0}$ we can write

$$
\begin{aligned}
& \left(\prod_{k=1}^{M} \omega\left(t_{M-k}^{\prime}, I_{M-k}^{\prime}\right)\right)^{-1} \prod_{j=1}^{N} \omega\left(t_{N-j}, I_{N-j}\right) \\
= & \left(\prod_{k=1}^{M} \omega\left(t_{M-k}^{\prime}, I_{M-k}^{\prime}\right)\right)^{-1}\left(\prod_{i=1}^{L} \omega\left(s_{L-i}, J_{L-i}\right)\right)^{-1} \prod_{i=1}^{L} \omega\left(s_{L-i}, J_{L-i}\right) \prod_{j=1}^{N} \omega\left(t_{N-j}, I_{N-j}\right) \\
= & \left(\prod_{i=1}^{L} \omega\left(s_{L-i}, J_{L-i}\right) \prod_{k=1}^{M} \omega\left(t_{M-k}^{\prime}, I_{M-k}^{\prime}\right)\right)^{-1} \prod_{i=1}^{L} \omega\left(s_{L-i}, J_{L-i}\right) \prod_{j=1}^{N} \omega\left(t_{N-j}, I_{N-j}\right) \\
= & \mathcal{P}\left(\omega, P_{*}^{T_{*}}\right)^{-1} \mathcal{P}\left(\omega, Q_{*}^{S_{*}}\right) \in U
\end{aligned}
$$


by our choice of $\gamma$. In view of our assumption that $G$ is complete, the existence of the total $\Pi_{a}^{c} \omega$ is now clear. Similarly we can show the existence of $\prod_{c}^{b} \omega$. From the obvious inclusion

$$
\mathcal{P}\left(\omega,\left.\gamma\right|_{[c, b]}\right) \mathcal{P}\left(\omega,\left.\gamma\right|_{[a, c]}\right) \subseteq \mathcal{P}\left(\omega,\left.\gamma\right|_{[a, b]}\right)
$$

the last identity follows by taking limits of both sides of this inclusion and making use of the continuity of multiplication in $G . \diamond$

The converse of this proposition does not require $G$ to be a topological group, but it requires $\omega$ to be atomless in the assumption. Let $G$ be a topological monoid. A $G$-valued integrator $\omega$ is said to be atomless if given any $c \in[a, b]$ and any basic neighbourhood $U \in \mathcal{N}_{G}$ there is a gauge $\eta$ such that $\omega(c, I) \in U$ and

$$
1 \in \omega(c, I) U \cap U \omega(c, I)
$$

whenever $(c, I)$ is a $\eta$-fine tagged interval in style $\mathcal{S}$; (recall that $a U=\{y=G \mid y=$ $a x$ for some $x \in U\}$ and $U a$ is clefined in the same manner). In the case that $G$ is a topological group, the additional condition $1 \in \omega(c, I) U \cap U \omega(c, I)$ is superfluous.

Proposition 3.6 Let $G$ be a topological monoid and $\omega$ be an atomless $G$-valued integrator, and $c \in(a, b)$. Suppose the restrictions $\omega_{1}=\left.\omega\right|_{[a, c]}$ and $\omega_{2}=\left.\omega\right|_{[c, b]}$ are both totalizable in style $\mathcal{S}$. Then $\omega$ is totalizable on all of $[a, b]$ and we have

$$
\prod_{a}^{b} \omega=\prod_{c}^{b} \omega \prod_{a}^{c} \omega
$$

Proof. It is enough to show that, for every gauge $\gamma_{1}$ of $[a, c]$ and any gauge $\gamma_{2}$ of $[c, b]$ and for every open neighbourhood $U$ of 1 , there is a gauge $\gamma$ on $[a, b]$ such that

$$
\mathcal{P}(\omega, \gamma) \subseteq \mathcal{P}\left(\left.\omega\right|_{[c, b]}, \gamma_{2}\right) U \mathcal{P}\left(\left.\omega\right|_{[a, c]}, \gamma_{1}\right)
$$

Let $V_{1}, V_{2}, V_{3}$ be open neighborhoods of 1 such that $V_{1} V_{2} V_{3} \subseteq U$. Take $\delta>0$ small enough so that if $(c, I)$ is $\delta$-fine, then $\omega(c, I) \in V_{2}, 1 \in \omega(c, I) V_{1}$ and $1 \in V_{3} \omega(c, I)$ which 
we can do since $\omega$ is atomless. Now define a gauge $\gamma$ on $[a, b]$ as follows: for $t \in[a, c)$ take $\gamma(t)=\min \left\{\gamma_{1}, c-t\right\}$, for $t \in(c, b]$ take $\gamma(t)=\min \left\{\gamma_{2}, t-c\right\}$, and

$$
\gamma(c)=\min \left\{\gamma_{1}(c), \gamma_{2}(c), \delta\right\}
$$

Notice that this gauge is "tag-forcing" at $c$ : if $P^{T}$ is a $\gamma$-fine tagged partition, then $c \in T$, that is, there is a tagged interval belonging to $P^{T}$ of the form $(c, I)$.

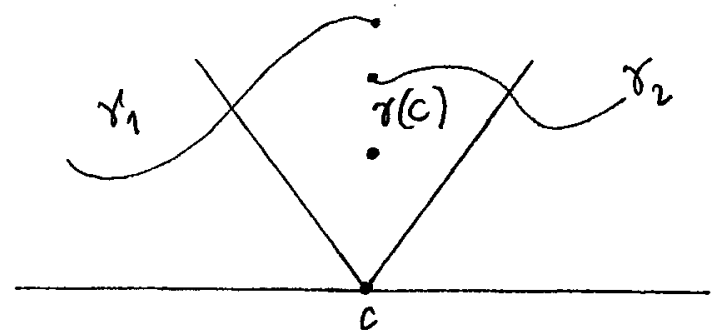

For any $\gamma$-fine tagged partition $P^{T}=\left\{\left(t_{k}, I_{k}\right)\right\}_{k=1}^{N}$ of $[a, b]$, consider the Riemann product

$$
\mathcal{P}\left(\omega, P^{T}\right)=\omega\left(t_{N}, I_{N}\right) \omega\left(t_{N-1}, I_{N-1}\right) \cdots \omega\left(t_{j}, I_{j}\right) \cdots \omega\left(t_{2}, I_{2}\right) \omega\left(t_{1}, I_{1}\right)
$$

with $t_{j}=c$. Take $J_{1}=I$ (and $J_{2}$ is absent) in case $c$ is the right endpoint of $I$ and $J_{2}=I$ (and $J_{1}$ is absent) in case $c$ is the left endpoint of $I$, and if it is in the interior of $I$, we let $J_{1}=I \cap[a, c]$ and $J_{2}=I \cap[c, b]$. Then we have

$$
\begin{aligned}
& \mathcal{P}\left(\omega, P^{T}\right) \in \omega\left(t_{N}, I_{N}\right) \cdots \omega\left(t_{j+1}, I_{j+1}\right) V_{2} \omega\left(t_{j-1}, I_{j-1}\right) \cdots \omega\left(t_{1}, I_{1}\right) \\
& \subseteq \omega\left(t_{N}, I_{N}\right) \cdots \omega\left(t_{j+1}, I_{j+1}\right)\left\{\omega\left(t_{j}, J_{1}\right) V_{1}\right\} V_{2}\left\{V_{3} \omega\left(t_{j}, J_{2}\right)\right\} \omega\left(t_{j-1}, I_{j-1}\right) \cdots \omega\left(t_{1}, I_{1}\right) \\
& \subseteq \omega\left(t_{N}, I_{N}\right) \cdots \omega\left(t_{j+1}, I_{j+1}\right) \omega\left(t_{j}, J_{1}\right) U \omega\left(t_{j}, J_{2}\right) \omega\left(t_{j-1}, I_{j-1}\right) \cdots \omega\left(t_{1}, I_{1}\right) \\
& \subseteq \mathcal{P}\left(\left.\omega\right|_{[c, b]}, \gamma_{2}\right) U \mathcal{P}\left(\left.\omega\right|_{[a, c]}, \gamma_{1}\right) .
\end{aligned}
$$

Now the proposition follows. $\diamond$ 
Given a monoid $G$ with multiplication $m: G \times G \rightarrow G$. The opposite monoid of $G$, denoted by $G^{\text {op }}$, is a monoid consisting of the set $G$ with the multiplication given by $(a, b) \mapsto m(b, a)$ for all $a, b \in G$. Notice that there is a natural isomorphism between $\left(G^{\mathrm{op}}\right)^{\mathrm{op}}$ and $G$ and hence we identify them. For convenience, for $a \in G$, we write $a^{\text {op }}$ for the corresponding element in $G^{\mathrm{op}}$. Then we have $(a b)^{\mathrm{op}}=b^{\mathrm{op}} a^{\mathrm{op}}$. The map from $G$ to $G^{\text {op }}$ sending $a$ to $a^{\text {op }}$ is an anti-isomorphism. In case $G$ is a group, $a \in G \mapsto a^{-1} \in G$ is an anti-automorphism of $G$ and hence $a \in G \rightarrow\left(a^{-1}\right)^{\text {op }} \in G^{\text {op }}$ is an isomorphism between $G$ and $G^{\text {op }}$. The following proposition is easy to establish:

Proposition 3.7 If $G$ is a topological group and if $\omega$ is a totalizable G-valued integrator, then the $G^{\mathrm{op}}$-valued integrator $\left(\omega^{-1}\right)^{\mathrm{op}}$ is also totalizable and $\left(\prod_{a}^{b} \omega\right)^{-1}=\left(\prod_{a}^{b}\left(\omega^{-1}\right)^{\mathrm{op}}\right)^{\mathrm{op}}$. Here $\left(\omega^{-1}\right)^{\mathrm{op}}$ is a $G^{\mathrm{op}}$-valued integrator given by $\left(\omega^{-1}\right)^{\mathrm{op}}(t, I)=\left[\omega(t, I)^{-1}\right]^{\mathrm{op}}$.

\subsection{Primitives and Hake's Theorem}

Let $G$ be a topological group. The primitive of a totalizable $G$-valued integrator $\omega$ is, by definition, the function $F:[a, b] \rightarrow G$ given by

$$
F(x)=\prod_{a}^{x} \omega .
$$

In the following proposition we assume that the topological monoid $G$ is regular, which means that every neighbourhood of a point $a$ in $G$ contains a closed neighbourhood of $a$.

Proposition 3.8 If $\omega$ is an atomless totalizable $G$-valued integrator in style $\mathcal{S}_{\mathrm{bd}}$, where $G$ is a regular topological monoid, then the primitive of $\omega$ is a continuous function.

Proof. First we show that $\lim _{x \uparrow b} F(x)=F(b)$. Take a neighbourhood $W_{b}$ of $F(b)$. Since $G$ is assumed to be regular, with no loss of generality, we assume that $W_{b}$ is closed. By the continuity of the multiplication $m: G \times G \rightarrow G$ at $(1, F(b))$, we can 
find a neighbourhood $U$ of 1 (i.e., $U \in \mathcal{N}_{G}$ ) and a neighbourhood $V_{b}$ of $F(b)$ such that $U V_{b} \subseteq W_{b}$. Since $\omega$ is totalizable on $[a, b]$, there is a gange $\gamma$ on $[a, b]$ such that $\mathcal{P}(\omega, \gamma) \subseteq V_{b}$. Also since $\omega$ is atomless, there is a gauge $\eta$ such that $1 \in U \omega(b, I)$ whenever $(b, I)$ is $\eta$-fine. Choose $\delta=\min \{\eta(b), \gamma(b)\}$ and let $x \in(b-\delta, b)$. We are going to show that $F(x) \in W_{b}$. Let $\gamma_{x}=\left.\gamma\right|_{[a, x]}$ and $\omega_{x}=\left.\omega\right|_{[a, x]}$, the restrictions of $\gamma$ and $\omega$ to $[a, x]$ respectively. Take any $\gamma_{x}$-fine tagged-partition $P^{T}=\left\{\left(t_{j}, I_{j}\right)\right\}_{j=1}^{N}$ of $[a, x]$. Then $\left\{\left(t_{j}, I_{j}\right)\right\}_{j=1}^{N+1}$ with $\left(t_{N+1}, I_{N+1}\right)=(b,[x, b])$ is a $\gamma$-fine tagged partition of $[a, b]$.

$$
\begin{aligned}
\mathcal{P}\left(\omega_{x}, P^{T}\right) & =\omega\left(t_{N}, I_{N}\right) \cdots \omega\left(t_{1}, I_{1}\right) \\
& \in\{U \omega(b,[x, b])\} \omega\left(t_{N}, I_{N}\right) \cdots \omega\left(t_{1}, I_{1}\right) \\
& =U \omega\left(t_{N+1}, I_{N+1}\right) \cdots \omega\left(t_{1}, I_{1}\right) \\
& \subseteq U V_{b} \subseteq W_{b}
\end{aligned}
$$

This shows $\Pi\left(\omega_{x}, \gamma_{x}\right) \subseteq W_{b}$. From Proposition 3.5 we know that the nest $\Pi\left(\omega_{x}\right)$ converges to $\Pi_{a}^{x} \omega \equiv F(x)$. Since $W_{b}$ is closed, we get $F(x) \in W_{b}$. The proof of $\lim _{x \downarrow b} F(x)=F(b)$ is similar and hence is omitted.

Next we establish the following version of Saks-Henstock's lemma:

Proposition 3.9 Let $\omega$ be a totalizable atomless $G$-valued integrator on $[a, b]$, where $G$ is a topological monoid with a regular topology. Then, for every neighbourhood $V$ of the total $\prod_{a}^{b} \omega$, there is a gauge $\gamma$ such that, whenever $\left\{\left(t_{j},\left[x_{2 j-1}, x_{2 j}\right]\right)\right\}_{j=1}^{m}$ is a $\gamma$-fine tagged figure (in an appropriate style), we have

$$
\left(\prod_{x_{2 m}}^{b} \omega\right) \omega\left(t_{m},\left[x_{2 m-1}, x_{2 m}\right]\right) \cdots\left(\prod_{x_{2}}^{x_{3}} \omega\right) \omega\left(t_{2},\left[x_{3}, x_{4}\right]\right)\left(\prod_{a}^{x_{1}} \omega\right) \omega\left(t_{1},\left[x_{1}, x_{2}\right]\right) \in V .
$$

(Here we agree that $\Pi_{c}^{c} \omega=1$.)

Proof. The proof of this proposition uses the same idea as that for the usual SaksHenstock's theorem and hence will be described only briefly. Since $G$ is regular, with no 
loss of generality we assume that $V$ is closed. From the totalizability of $\omega$ we know that there exists a gauge $\gamma$ on $[a, b]$ such that $\Pi(\omega, \gamma) \subseteq V$. Let $\left\{\left(t_{j},\left[x_{2 j-1}, x_{2 j}\right]\right\}_{j=1}^{m}\right.$ be a $\gamma$-fine tagged figure in $[a, b]$. If, on each of the complemented intervals

$$
\left[a, x_{1}\right],\left[x_{2}, x_{3}\right], \ldots,\left[x_{2 j-2}, x_{2 j-1}\right] \quad\left[x_{2 j}, b\right]
$$

we take a $\gamma$-fine tagged partition, then the tagged figure of $[a, b]$, together with these tagged partitions, form a $\gamma$-fine tagged partition of $[a, b]$. Thus we have

$$
\begin{aligned}
& \Pi\left(\left.\omega\right|_{\left[x_{2 m}, b\right]},\left.\gamma\right|_{\left[x_{2 m}, b\right]}\right) \omega\left(t_{n},\left[x_{2 m-1}, x_{2 m}\right]\right) \cdots \\
& \quad \cdots \omega\left(t_{2},\left[x_{3}, x_{4}\right]\right) \Pi\left(\left.\omega\right|_{\left[x_{2}, x_{3}\right]},\left.\gamma\right|_{\left[x_{2}, x_{3}\right]}\right) \omega\left(t_{1},\left[x_{1}, x_{2}\right]\right) \Pi\left(\left.\omega\right|_{\left[a, x_{1}\right]},\left.\gamma\right|_{\left[a, x_{1}\right]}\right) \subseteq V .
\end{aligned}
$$

Notice that $\prod_{a}^{x_{1}} \omega$ is in the closure of the set $\mathcal{P}\left(\left.\omega\right|_{\left[a, x_{1}\right]},\left.\gamma\right|_{\left[a, x_{1}\right]}\right)$, and $\prod_{x_{2}}^{x_{3}} \omega$ is in the closure of the set $\mathcal{P}\left(\left.\omega\right|_{\left[x_{2}, x_{3}\right\}},\left.\gamma\right|_{\left\{x_{2}, x_{3}\right]}\right)$, etc. Now the theorem is clear.

Corollary 3.10 Let $G$ be a topological group and let $\omega$ be a totalizable atomless $G$-valued integrator over $[a, b]$. Then, for each neighbourhood $V$ of the identity, there is a gauge on $[a, b]$ such that, for each $c \in(a, b)$ and each $\gamma$-fine partition $P^{T}$ of $[a, c]$, we have

$$
\mathcal{P}\left(\left.\omega\right|_{[a, c]}, P^{T}\right) \subseteq\left(\prod_{a}^{c} \omega\right) V
$$

Proof. By the proposition we can find a gauge $\gamma$ on $[a, b]$ such that, for all $c \in(a, b)$ and for all $\gamma$-fine tagged partitions $P^{T}$ of $[a, c]$,

$$
\left(\prod_{c}^{b} \omega\right) \mathcal{P}\left(\left.\omega\right|_{[a, c]}, P^{T}\right) \subseteq\left(\prod_{a}^{b} \omega\right) V
$$

Multiplying both sides on the left by $\left(\Pi_{c}^{b} \omega\right)^{-1}$, we get the desired inclusion relation. $\diamond$

Now we can prove a version of Hake's theorem for product integrals in the bound style. The proof here is a modification of the standard proof (which is already rather complicated, see, e.g. [Pfe]) of this theorem, with extra complications due to the noncommutativity of multiplication. 
THEOREM 3.11 Let $G$ be a topological group and let $\omega$ be an atomless $G$-valued integrator on $[a, b]$ such that $F(x)=\prod_{a}^{x} \omega$ exists for all $x \in(a, b)$. Suppose that $p \equiv \lim _{x \uparrow b} F(x)$ exists. Then $\omega$ is totalizable and $\Pi_{a}^{b} \omega=p$.

Proof. We extend $F$ to a continuous function on $[a, b]$ by putting $F(b)=p$. Let $W$ be a given neighbourhood of 1 . Take any neighbourhood $U$ such that $U U U \subseteq W$. By Lemma 3.1 , we can find a sequence $\left\{U_{n}\right\}$ of neighbourhood of 1 such that $U_{n} U_{n-1} \cdots U_{2} U_{1} \subseteq U$ for all $n$. Take a strictly increasing sequence $\left\{c_{n}\right\}_{n \geq 1}$ in $[a, b]$ such that $c_{1}=a$ and $\lim _{n \rightarrow \infty} c_{n}=b$. By Proposition 3.5 we know that $\omega$ is totalizable on each $\left[c_{n}, c_{n+1}\right]$. For each $k$, we can take a closed neighbourhood $V_{k}$ of 1 such that

$$
V_{k} V_{k}\left(\prod_{a}^{c_{k}} \omega\right) F(b)^{-1} \subseteq\left(\prod_{a}^{c_{k}} \omega\right) F(b)^{-1} U_{k}
$$

Denote by $\omega_{k}$ the restriction of $\omega$ to $\left[c_{k}, c_{k+1}\right]$. Let $\gamma_{k}$ be a gauge on $\left[c_{k}, c_{k+1}\right]$ such that

$$
\prod\left(\omega_{k}, \gamma_{k}\right)\left(\prod_{c_{k}}^{c_{k+1}} \omega\right)^{-1} \subseteq V_{k+1}
$$

Let $\gamma$ be a gauge on $[a, b]$ such that

1. $\left.\gamma\right|_{\left[c_{n}, c_{n+1}\right]} \leq \gamma_{n}$ for each $n$.

2. $\omega(b,[u, b]) \in U$ and $\left(\Pi_{a}^{u} \omega\right) F(b)^{-1} \in U$ if $(b,[u, b])$ is $\gamma$-fine.

3. $\omega\left(c_{n}, I_{1}\right)^{-1} \omega\left(c_{n}, I_{2}\right) \omega\left(c_{n}, I_{3}\right)^{-1} \in V_{n}$ if $\left(c_{n}, I_{1}\right),\left(c_{n}, I_{2}\right),\left(c_{n}, I_{3}\right)$ are arbitrary $\gamma$-fine tagged intervals with $c_{n}$ as their common tag.

4. $\gamma \leq \Gamma$, where $\Gamma$ is a "tagged forcing" gauge depicted on the next page.

Condition 4 entails: (a) for each $x \in\left(c_{n}, c_{n+1}\right)$,

$$
\gamma(x) \leq \min \left\{x-c_{n}, c_{n+1}-x\right\}
$$

(b) $\gamma\left(c_{n}\right)<\frac{1}{2} \min \left\{c_{n+1}-c_{n}, c_{n}-c_{n-1}\right\}$ and (c) $\gamma(x)<b-x$ for all $x \in[a, b)$.

Let $P^{T}=\left\{\left(t_{j},\left[x_{j-1}, x_{j}\right]\right\}_{j=1}^{N}\right.$ be a $\gamma$-fine tagged partition of $[a, b]$. By condition (c), we know that $t_{N}=b$. Let $M$ be the positive integer such that $c_{M} \leq x_{N-1}<c_{M+1}$. (In 
the following argeument we assume $c_{M}<x_{N-1}$. When $c_{M}=x_{N-1}$, the argument can be simplified.) By condition (a) we know that if $t_{j}$ is a tag in an open interval $\left(c_{m} \cdot c_{m+1}\right)$ for some $m$, then the corresponding interval $\left[x_{j}, x_{j+1}\right]$ is contained in $\left(c_{m}, c_{m+1}\right)$. Condition (b) tells us that if $t_{j}=c_{m}$ for some $m$, then $c_{m}$ is the only point from the sequence $\left\{c_{n}\right\}_{n \geq 1}$ contained in $\left[x_{j}, x_{j+1}\right]$. Now it is clear that all $c_{1}, c_{2}, \ldots, c_{M}$ must be among the tags $\left\{t_{1}, t_{2}, \ldots, t_{N}\right\}$.

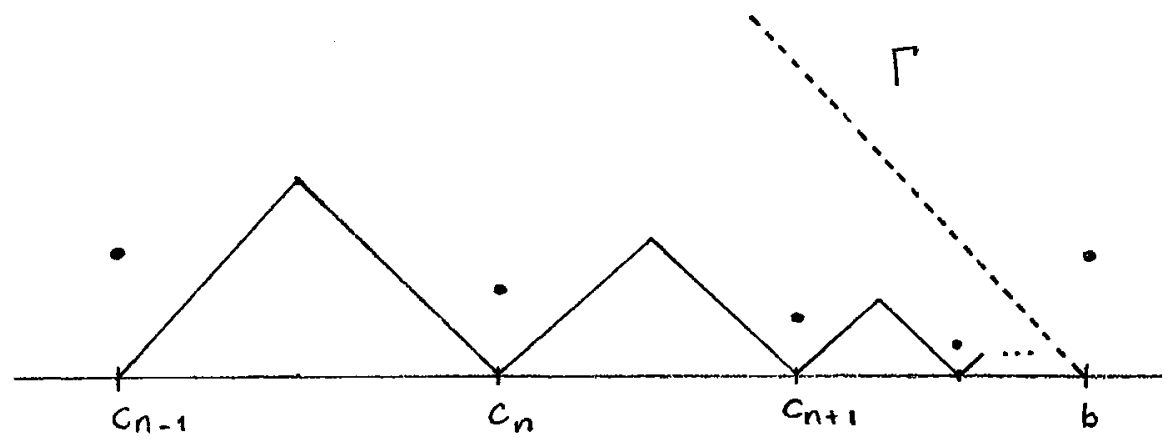

Now we adjust the Riemann product $\mathcal{P}\left(\omega, P^{T}\right)$ as follows: at each $c_{k}$, say $c_{k}=t_{j}$, replace the factor $\omega\left(t_{j},\left[x_{j-1}, x_{j}\right]\right)$ by the product $\omega\left(t_{j},\left[t_{j}, x_{j}\right]\right) \omega\left(t_{j},\left[x_{j-1}, t_{j}\right]\right)$. In this way we change $\mathcal{P}\left(\omega, P^{T}\right)$ into a new Riemann product which can be written as

$$
\omega\left(b,\left[x_{N-1}, x_{N}\right]\right) \mathcal{P}\left(\left.\omega\right|_{\left\{c_{M}, x_{N-1}\right]}, P_{M}^{T_{M}}\right) \mathcal{P}\left(\omega_{M-1}, P_{M-1}^{T_{M-1}}\right) \cdots \mathcal{P}\left(\omega_{1}, P_{1}^{T_{1}}\right)
$$

where each $P_{j}^{T_{j}}$ is a $\gamma$-fine tagged partition of an appropriate subinterval. Condition 3. tells us the discrepancy that arises in this modification:

$$
\omega\left(t_{j},\left[x_{j-1}, x_{j}\right]\right) \in \omega\left(t_{j},\left[t_{j}, x_{j}\right]\right) V_{j} \omega\left(t_{j},\left[x_{j-1}, t_{j}\right]\right)
$$

hence we have

$$
\mathcal{P}\left(\omega, P^{T}\right) \in \omega\left(b,\left[x_{N-1}, b\right]\right) \mathcal{P}\left(\left.\omega\right|_{\left.\left[c_{M}, x_{N-1}\right)\right]}, P_{M}^{T_{M}}\right) V_{M} \cdots \mathcal{P}\left(\omega_{2}, P_{2}^{T_{2}}\right) V_{2} \mathcal{P}\left(\omega_{1}, P_{1}^{T_{1}}\right)
$$

By 3.3, we have $\mathcal{P}\left(\omega_{k}, P_{k}^{T_{k}}\right) \in V_{k+1}\left(\prod_{c_{k}}^{c_{k+1}} \omega\right)$. So 


$$
\begin{array}{rl}
V_{M} & \mathcal{P}\left(\omega_{M-1}, P_{M-1}^{T_{M-1}}\right) V_{M-1} \cdots V_{3} \mathcal{P}\left(\omega_{2}, P_{2}^{T_{2}}\right) V_{2} \mathcal{P}\left(\omega_{1}, P_{1}^{T_{1}}\right) F(b)^{-1} \\
& \subseteq V_{M} \mathcal{P}\left(\omega_{M-1}, P_{M-1}^{T_{M-1}}\right) V_{M-1} \cdots V_{3} \mathcal{P}\left(\omega_{2}, P_{2}^{T_{2}}\right) V_{2} V_{2}\left(\prod_{c_{1}}^{c_{2}} \omega\right) F(b)^{-1} \\
& \subseteq V_{M} \mathcal{P}\left(\omega_{M-1}, P_{M-1}^{T_{M-1}}\right) V_{M-1} \cdots V_{3} \mathcal{P}\left(\omega_{2}, P_{2}^{T_{2}}\right)\left(\prod_{c_{1}}^{c_{2}} \omega\right) F(b)^{-1} U_{1} \\
& \subseteq V_{M} \mathcal{P}\left(\omega_{M-1}, P_{M-1}^{T_{M-1}}\right) V_{M-1} \cdots V_{3} V_{3}\left(\prod_{c_{2}}^{c_{3}} \omega \prod_{c_{1}}^{c_{3}} \omega\right) F(b)^{-1} U_{1} \\
& \subseteq V_{M} \mathcal{P}\left(\omega_{M-1}, P_{M-1}^{T_{M-1}}\right) V_{M-1} \cdots\left(\prod_{c_{1}}^{c_{3}} \omega\right) F(b)^{-1} U_{2} U_{1} \\
& \subseteq \cdots \cdots \subseteq\left(\prod_{c_{1}}^{c_{M}} \omega\right) F(b)^{-1}\left\{U_{M} \cdots U_{2} U_{1}\right\} .
\end{array}
$$

From the proof of Corollary 3.10, we see that

$$
\mathcal{P}\left(\left.\omega\right|_{\left[c_{M}, x_{N-1}\right]}, P_{M}^{T_{M}}\right) \subseteq V_{M+1}\left(\prod_{c_{M}}^{x_{N-1}} \omega\right)
$$

(notice that $\left[c_{M}, x_{N-1}\right]$ is a subinterval of $\left[c_{M}, c_{M+1}\right]$ and $V_{M+1}$ is assumed to be closed.) Thus we arrive at

$$
\left.\mathcal{P}\left(\omega, P^{T}\right) F(b)^{-1} \in \omega\left(b,\left[x_{N-1}, x_{N}\right]\right)\left(\prod_{a}^{x_{N-1}} \omega\right)\right) F(b)^{-1}\left\{U_{M+1} U_{M} \cdots U_{2} U_{1}\right\} \subseteq U U U \subseteq W .
$$

Hence $\omega$ is totalizable. $\diamond$

\subsection{Integrators With Values in the Group of Invertible Elements in a Banach Algebra}

Let $A$ be a Banach algebra with identity element 1 . The group of all invertible elements in $A$ will be denoted by $G \equiv G(A)$. Then $G$ is an open set in $A$, as well as each of its (connected) components. Denote by $G_{0}$ the component containing the identity 1 . The exponential function 
$\exp : A \rightarrow G_{0}$ is given by

$$
\exp x=\sum_{n=0}^{\infty} \frac{1}{n !} x^{n} \quad \text { (which is absolutely convergent). }
$$

A well-known fact is that $G_{0}$ is an open subgroup of $G$ and it is the group generated by $\exp (A)$, the range of $\exp$. Notice that $\exp (A)$ contains the open ball $B(1 ; 1)$ of radius 1 centered at 1 . In fact, if we define the principal logarithm on $B(1 ; 1)$ by putting

$$
\log (1-x)=-x-\frac{x^{2}}{2}-\frac{x^{3}}{3}-\cdots
$$

for all $x \in A$ with $\|x\|<1$, then we have exp $\log (1-x)=1-x$. The image $V \equiv \log (B(1 ; 1))$ of the open ball $B(1 ; 1)$ via the $\log$ function is an open set in $A$ and the exponential map restricted to $V$ gives a homeomorphism between $V$ and $B(1 ; 1)$. In case $A$ is commutative, then we simply have $G_{0}=\exp (A)$.

Now we consider an atomless totalizable integrator $\omega$ on $[a, b]$ with values in $G$. Consider the primitive $F:[a, b] \rightarrow A$ of $\omega$ given by $F(x)=\Pi_{a}^{x} \omega$. Then $F(a)=1$ and by Proposition 3.8 above we know that $F$ is continuous. By the connectedness of $G_{0}$, we see that $F(x) \in G_{0}$ for all $x \in[a, b]$. Using a compactness argument, we see that there exists $\delta>0$ such that $\prod_{u}^{v} \omega \in B(1 ; 1)$ for all $u, v \in[a, b]$ with $u<v$ and $v-u<\delta$. Thus, with no loss of generality, we assume that $F(x) \in B(1,1)$ for all $x \in[a, b]$. Since $\omega$ is atomless, for each $t \in[a, b]$, there exists $\eta>0$ such that for all intervals so that, for all $(t, I) \in \mathcal{S}$ with $I \subseteq(t-\eta, t+\eta)$, we have $\omega(t, I) \in B(1 ; 1)$. In other words, there is a gauge $\gamma_{0}$ on $[a, b]$ such that $\omega(t, I) \in B(1 ; 1)$ for all $\gamma_{0}$-fine tagged intervals $(t, I)$ of style $\mathcal{S}$. So, with no loss of generality, we assume that $\omega(t, I) \in B(1 ; 1)$ for all $(t, I) \in \mathcal{S}$. In case the Banach algebra $A$ is commutative, the product integration is well-understood:

Proposition 3.12 Let $\omega$ be an atomless integrator on $[a, b]$ in style $\mathcal{S}$ with values in the group $G$ of invertible elements in a commutative Banach algebra $A$. Then the product 
integral $\Pi_{a}^{b} \omega$ exists if and only if there is an additive totalizable integrator $\alpha$ on $[a, b]$ in style $\mathcal{S}$ with values in $A$ such that $\omega=\exp \alpha$, that is,

$$
\omega(t, I)=e^{\alpha(t, I)} \quad \text { for all }(t, I) \in \mathcal{S}
$$

Furthermore, in that case we have $\Pi_{a}^{b} \omega=\exp \int_{a}^{b} \alpha$.

Proof. Let $\gamma_{0}$ be a gauge on $[a, b]$ such that $\omega(t, I) \in B(1 ; 1)$ for all $\gamma_{0}$-fine tagged interval $(t, I) \in \mathcal{S}$. Define an integrator $\alpha: \mathcal{S} \rightarrow A$ by putting $\alpha(t, I)=\log \omega(t, I)$ if $(t, I)$ is $\gamma_{0}$-fine and $\alpha(t, I)=0$ otherwise. Then, for every gauge $\gamma$ on $[a, b]$ with $\gamma \leq \gamma_{0}$ and every $\gamma$-fine partition $P^{T} \equiv\left\{\left(t_{j}, I_{j}\right)\right\}_{j=1}^{N}$, we have

$$
\mathcal{P}\left(\omega, P^{T}\right)=\prod_{j=1}^{N} \omega\left(t_{j}, I_{j}\right)=\prod_{j=1}^{N} \exp \alpha\left(t_{j}, I_{j}\right)=\exp \left(\sum_{j=1}^{N} \alpha\left(t_{j}, I_{j}\right)\right) .
$$

Now the proposition is clear.

The above proposition suggests to us to consider gauge product integrals either of the form $\prod_{a}^{b}(1+\alpha)$ or $\prod_{a}^{b} e^{\alpha}$, where $\alpha$ is an additive integrator. Our final result shows that, under some general assumption, they are equivalent. Here, we drop the assumption that $A$ is commutative. First we give some definitions: we say that an integrator $\alpha: \mathcal{S} \rightarrow A$ has a vanishing second variation if, given any $\varepsilon>0$, there exists a gauge $\gamma$ on $[a, b]$ such that

$$
\sum_{k=1}^{N}\left\|\alpha\left(t_{k}, I_{k}\right)\right\|^{2}<\varepsilon
$$

for all $\gamma$-fine tagged partition $\left\{\left(t_{k}, I_{k}\right)\right\}_{k=1}^{N}$ of $[a, b]$ in style $\mathcal{S}$. We say that a multiplicative integrator $\omega: \mathcal{S} \rightarrow A$ is bounded if there exists $M>0$ and a gauge $\gamma_{0}$ on $[a, b]$ such that $\left\|\mathcal{P}\left(\omega, P^{T}\right)\right\| \leq M$ for all $\gamma_{0}$-fine partition $P^{T}$ of any subinterval $[c, d]$ of $[a, b]$ in style $\mathcal{S}$.

Proposition 3.13 If $\alpha: \mathcal{S} \rightarrow A$ is an additive integrator of style $\mathcal{S}$ on $[a, b]$ with a vanishing second variation and if $1+\alpha$ and $e^{\alpha}$ are bounded multiplicative integrators, then the product integral $\Pi_{a}^{b}(1+\alpha)$ exists if and only if the product integral $\prod_{a}^{b} e^{\alpha}$ exists, and they are equal if they exist. 
Proof. Let $\gamma_{0}$ be a gauge such that $\gamma_{0}<1$ and for each $\gamma_{0}$-fine tagged partition $P^{T}$ of a subinterval of $[a, b]$, we have $\left\|\mathcal{P}\left(1+\alpha, P^{T}\right)\right\| \leq M$ and $\left\|\mathcal{P}\left(e^{\alpha}, P^{T}\right)\right\| \leq M$. Take a $\gamma_{0}$-fine partition $P^{T}=\left\{\left(t_{k}, I_{k}\right)\right\}_{k=1}^{n}$ of $[a, b]$. We can rewrite

$$
\left\|\mathcal{P}\left(e^{\alpha}, P^{T}\right)-\mathcal{P}\left(1+\alpha, P^{T}\right)\right\|=\left\|\prod_{k=1}^{n} e^{\alpha\left(t_{k}, I_{k}\right)}-\prod_{k=1}^{n}\left(1+\alpha\left(t_{k}, I_{k}\right)\right)\right\|
$$

as a telescoping sum

$$
\| \sum_{k=1}^{n}\left(\prod_{j<k}\left(1+\alpha\left(t_{j}, I_{j}\right)\right)\left(e^{\alpha\left(t_{k}, I_{k}\right)}-1-\alpha\left(t_{k} . I_{k}\right)\right)\left(\prod_{j>k} e^{\alpha\left(t_{j}, I_{j}\right)}\right) \|\right.
$$

Note that, for all $a \in A$ with $\|a\|<1$,

$$
\left\|e^{a}-1-a\right\|=\left\|\frac{a^{2}}{2 !}+\frac{a^{3}}{3 !}+\cdots\right\| \leq \frac{\|a\|^{2}}{2}+\frac{\|a\|^{2}}{2^{2}}+\cdots \leq\|a\|^{2} .
$$

Hence we have the estimate

$$
\left\|\mathcal{P}\left(e^{\alpha}, P^{T}\right)-\mathcal{P}\left(1+\alpha, P^{T}\right)\right\| \leq M^{2} \sum_{k=1}^{n}\left\|e^{\alpha\left(t_{k}, I_{k}\right)}-1-\alpha\left(t_{k}, I_{k}\right)\right\| \leq M^{2} \sum_{k=1}^{n}\left\|\alpha\left(t_{k}, I_{k}\right)\right\|^{2}
$$

The rest is clear. $\diamond$ 


\section{References}

[Bar] R. G. Bartle, Return to the Riemann integral, the Amer. Math. Monthly, Math. Asso. of Amer., 103, (1996), 625-632.

[Day] M. M. Day, Normed Linear Spaces, Academic Press Inc., Publishers, New York, 1962.

[Die] J. Diestel, Sequences and Series in Banach Spaces, Springer-Verlag, New York, 1984.

[DiU] J. Diestel and J. J. Uhl, Jr., Vector Measures, Mathematical Surveys, No. 15, Amer. Math. Soc. Providence, 1977.

[Fo1] C.K. Fong, A Continuous Version of Orlicz-Pettis Theorem Via Vector-Valued HenstockKurzweil Integrals, Czech Math. Jour., 52 (2002) 531-536.

[Fo2] C.K. Fong, Notes on Gauge Integration, 2000 (unpublished).

[GoK] I.C. Gohberg and M.G. Krein, Theory and Applications of Volterra Operators in Hilbert Space, Translations of mathmatical Monographs, Vol. 24, Amer. Math. Soc., Providence, Rhode Island, 1970.

[Gor] R.A. Gordan The Integrals of Lebesgue, Denjoy, Perron and Henstock, Graduate Studies in Mathematics, Amer. Math. Soc. (1994)

[Hen] R. Henstock, The General Theory of Integration, Clarendon Press, Oxford, 1991.

[Kur] J. Kurzweil, Generalized Ordinary Differential Equations and Continuous Dependence on a parameter, Czech. Math. Jour., 7 (1957), 418-449.

[KuJ] J. Kurzweil and J. Jarnik, A Perron Integral, Perron Product Integral and Ordinary Linear Differential Equations, Springer's Lecture Notes in Math., 1192 (1986), 149154.

[Le1] S. Leader, The Kurzweil-Henstock Integral and Its Differentials, Marcel Dekkar, Inc. New York, 2001.

[Le2] S. Leader, What Is a Differential? A New Answer From the generalized Riemann Integral, Amer. Math. Monthly, 93, (1986), 348-356.

[McS] E.J. McShane, A Riemann Type Integral that Includes Lebesgue--Stieltjes, Bochner and Stochastic Integrals, Memoirs, Amer. Math. Soc. 88, 1969. 
[Oks] B. Oksendal, Stochastic Differential Equations, Sixth edition, Univeritext, Springer Verlag, Berlin, 2003,

[Pes] I.N. Pesin,Classical and Modern Integration Theories. translated from Russian and edited by S. Kotz, Academic Press, New York, 1970.

[Pfe] W. F. Pfeffer, The Riemann Approach to Integration, Cambridge Tracts in Math., No. 109, Cambridge University Press, Cambridge, 1993.

[Rog] C.A. Rogers, Hausdorff Measures Cambridge University Press, Cambridge, 1970.

[Sak] S. Saks, Theory of the Integral, Second Revised Edition, Translated from Polish by L.C. Young, Hafner Publ. Co., New York, 1937.

[Ste] E. M. Stein, Singular Integrals and Differentiability Properties of Functions, Princeton University Press, Princeton, 1970. 